\title{
The Suprathermal Ion Telescope (SIT) for the IMPACT/SEP Investigation
}

\author{
G. M. Mason · A. Korth · P. H. Walpole · M. I. Desai • \\ T. T. Von Rosenvinge $\cdot$ S. A. Shuman
}

Received: 5 July 2006 / Accepted: 25 October 2006 / Published online: 27 April 2007

(C) Springer Science + Business Media B.V. 2007

\begin{abstract}
The Solar-Terrestrial Relations Observatory (STEREO) mission addresses critical problems of the physics of explosive disturbances in the solar corona, and their propagation and interactions in the interplanetary medium between the Sun and Earth. The InSitu-Measurements of Particles and CME Transients (IMPACT) investigation observes the consequences of these disturbances and other transients at $1 \mathrm{AU}$. The generation of energetic particles is a fundamentally important feature of shock-associated Coronal Mass Ejections (CMEs) and other transients in the interplanetary medium. Multiple sensors within the IMPACT suite measure the particle population from energies just above the solar wind up to hundreds of MeV/nucleon. This paper describes a portion of the IMPACT Solar Energetic Particles (SEP) package, the Suprathermal Ion Telescope (SIT) which identifies the heavy ion composition from the suprathermal through the energetic particle range ( $\sim$ few $10 \mathrm{~s}$ of $\mathrm{keV} /$ nucleon to several $\mathrm{MeV} /$ nucleon). SIT will trace and identify processes that energize low energy ions, and characterize their transport in the interplanetary medium. SIT is a timeof-flight mass spectrometer with high sensitivity designed to derive detailed multi-species particle spectra with a cadence of $60 \mathrm{~s}$, thereby enabling detailed studies of shock-accelerated and other energetic particle populations observed at $1 \mathrm{AU}$.
\end{abstract}

Keywords Solar energetic particles (SEPs) $\cdot$ Cosmic rays $\cdot$ Shock acceleration $\cdot$ CMEs

G. M. Mason ( $₫)$

Johns Hopkins University/Applied Physics Laboratory, Laurel, MD 20723, USA

e-mail: glenn.mason@jhuapl.edu

A. Korth

Max-Planck-Institut für Sonnensystemforschung, Lindau, Germany

P. H. Walpole

University of Maryland, College Park, MD 20742

M. I. Desai

Southwest Research Institute, San Antonio, TX 78238

T. T. Von Rosenvinge $\cdot$ S. A. Shuman

NASA/Goddard Spaceflight Center, Greenbelt, MD 20771 


\section{Scientific Goals}

\subsection{Particle Acceleration Near the Sun and in the Heliosphere}

The production of energetic particles takes place throughout the heliosphere with multiple processes taking place at different locations and times. In interplanetary space at $1 \mathrm{AU}$, this wide variety of sources is sketched in Figure 1, which shows a multi-year sum of the $\mathrm{O}$ fluence over the energy range $<1 \mathrm{keV} /$ nucleon to $>100 \mathrm{MeV} /$ nucleon (adapted from Mewaldt et al., 2001). Starting at the low energy end we have the solar wind plasma, whose characteristic flow speed is generally either "slow" ( $\sim 400 \mathrm{~km} / \mathrm{s})$ or "fast" ( $\sim 700 \mathrm{~km} / \mathrm{s})$. Above the range dominated by the solar wind there is a large decrease in intensity leading to

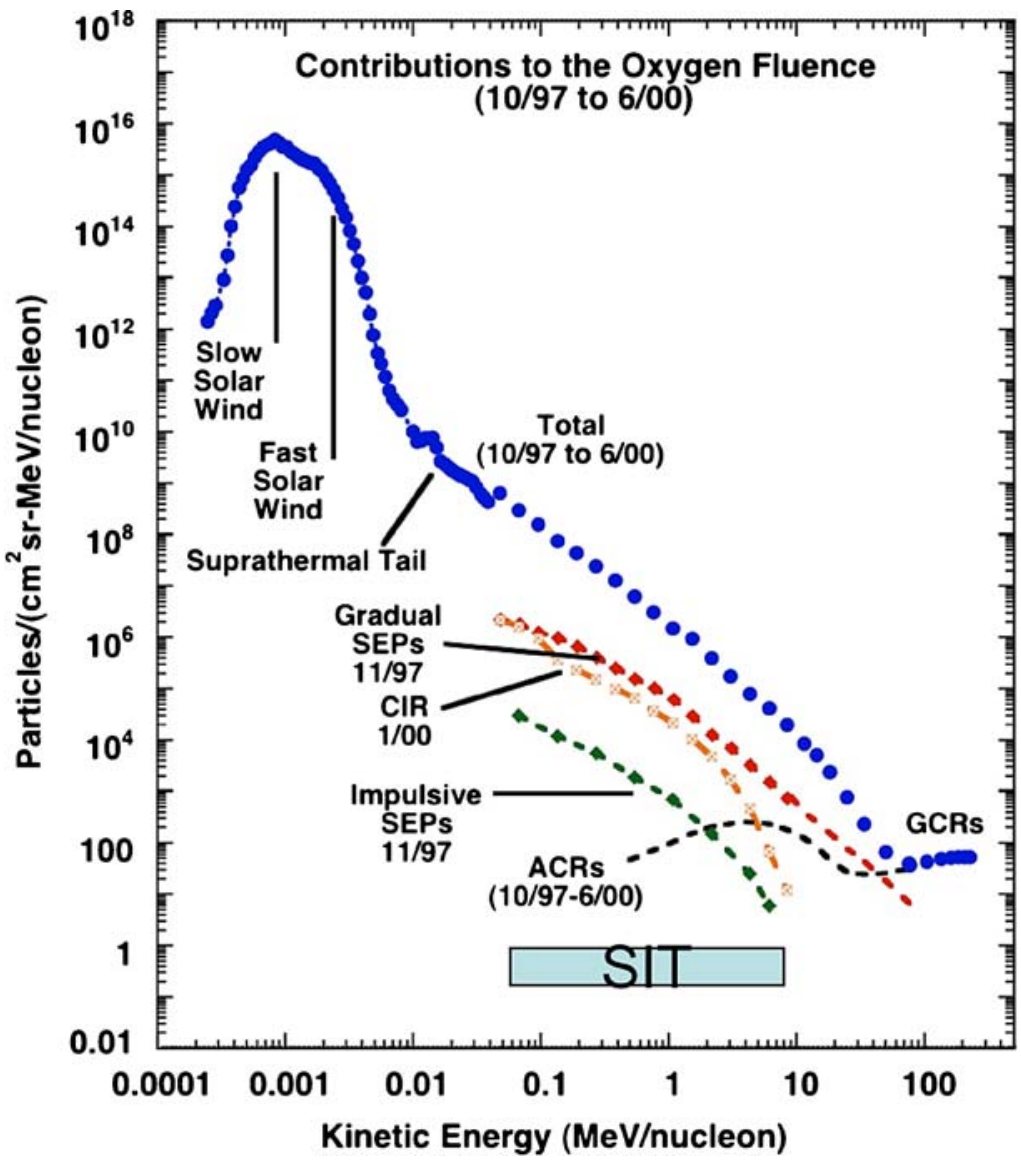

Fig. 1 Long term fluence of O summed from several instruments on the $A C E$ spacecraft from Oct. 1997 through June 2000. In addition to the total fluence (filled blue circles) the figure also shows typical contributions from a Gradual Solar Energetic Particle (SEP) event, a typical Corotating Interaction Region (CIR) event, and a typical Impulsive SEP event. Also shown are the long term average levels of Anomalous Cosmic Rays (ACRs) during quiet periods during the summing interval. At the highest energies, the falling spectrum flattens and rises slowly due to the Galactic Cosmic Rays (GCRs). Shaded box shows the energy coverage of the SIT sensor (figure adapted from Mewaldt et al., 2001) 


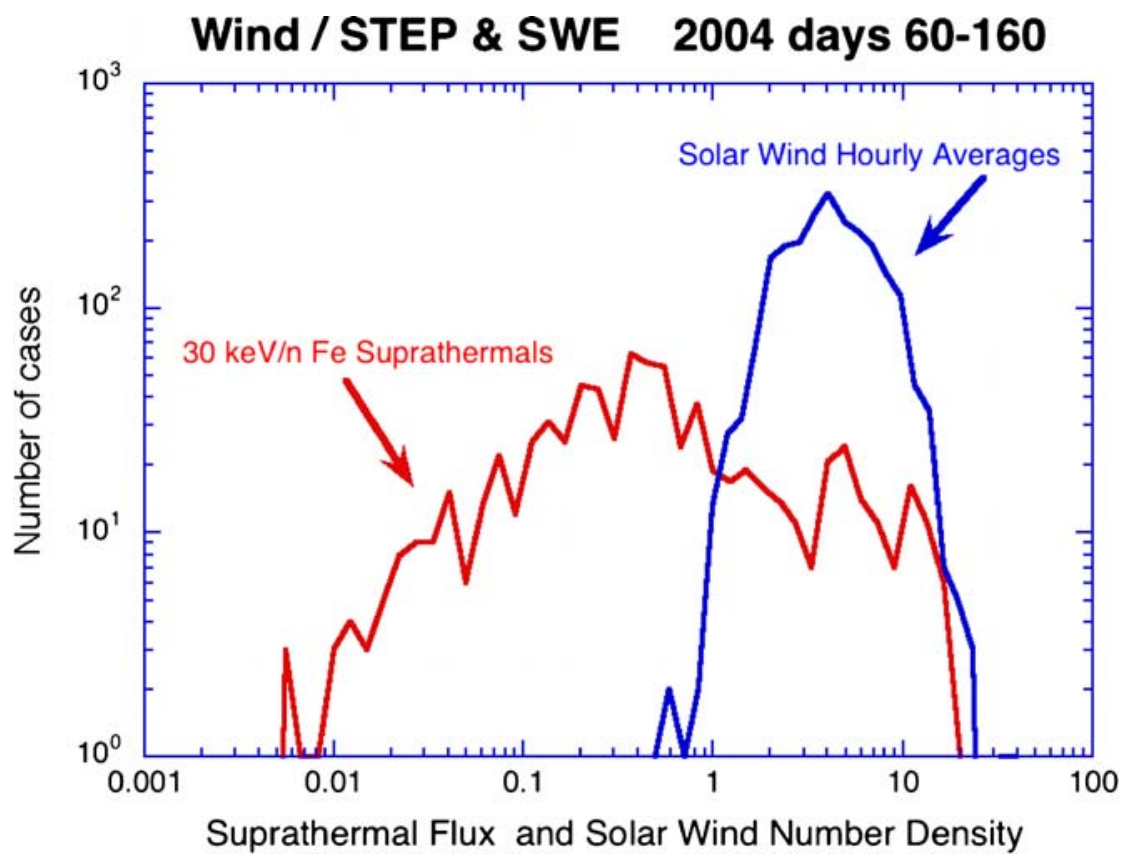

Fig. 2 Hourly average suprathermal (red) and solar wind (blue) flux values for a 100 day period in 2004. While the solar wind shows a factor of 10 variation, the $30 \mathrm{keV} /$ nuc Fe suprathermals show a factor of 1000 variation (from Mason et al., 2005)

a suprathermal tail that extends from several times the solar wind speed to $\sim 10 \mathrm{MeV} /$ nucleon, where particle speeds are $\sim 0.15 c$, about 100 times the solar wind speed. In this energy range of $\sim 0.01$ to $10 \mathrm{~s}$ of $\mathrm{MeV} /$ nucleon, multiple particle sources exist, with each dominating the intensities at one time or another. The largest of these are gradual Solar Energetic Particle (SEP) events, which last for days and occur with a frequency of $\sim 1 /$ month during active periods of the solar cycle. At generally lower intensities are Corotating Interaction Regions (CIRs) which are generally most intense during the declining phase of the sunspot cycle, and whose primary acceleration region and maximum intensities occur at a few AU. At even lower levels are impulsive SEPs, brief events with enhanced electron, ${ }^{3} \mathrm{He}$, and heavy ion abundances (for a recent review, see Reames, 1999). At energies above $\sim 10 \mathrm{MeV} /$ nucleon Anomalous Cosmic Rays (ACRs) and Galactic Cosmic Rays (GCRs) dominate the average intensities.

In the last few years it has become apparent that interplanetary suprathermal particles are a key component of the seed population of particles energized in gradual SEP events and by traveling interplanetary shocks (e.g., Mason et al., 1999a, 2005; Desai et al., 2001, 2005, 2006; Tylka et al., 2001, 2003, 2006a; Mewaldt et al., 2006). It can be seen from Figure 1 that this suprathermal tail has many contributors including heated solar wind, material from prior large SEP events, impulsive SEP events, traveling shock events, etc. This leads to a much larger variability than is observed in the solar wind itself. For example, Figure 2 shows the hourly averaged intensities of Fe suprathermals and solar wind over a 100 day period in 2004 . Note that while the solar wind shows a factor of $\sim 10$ variation in density, the suprathermals show a factor of $\sim 1000$. Thus, if the seed particles energized by interplanetary shocks are solely suprathermals, then the range of input densities the shocks encounter will be much 


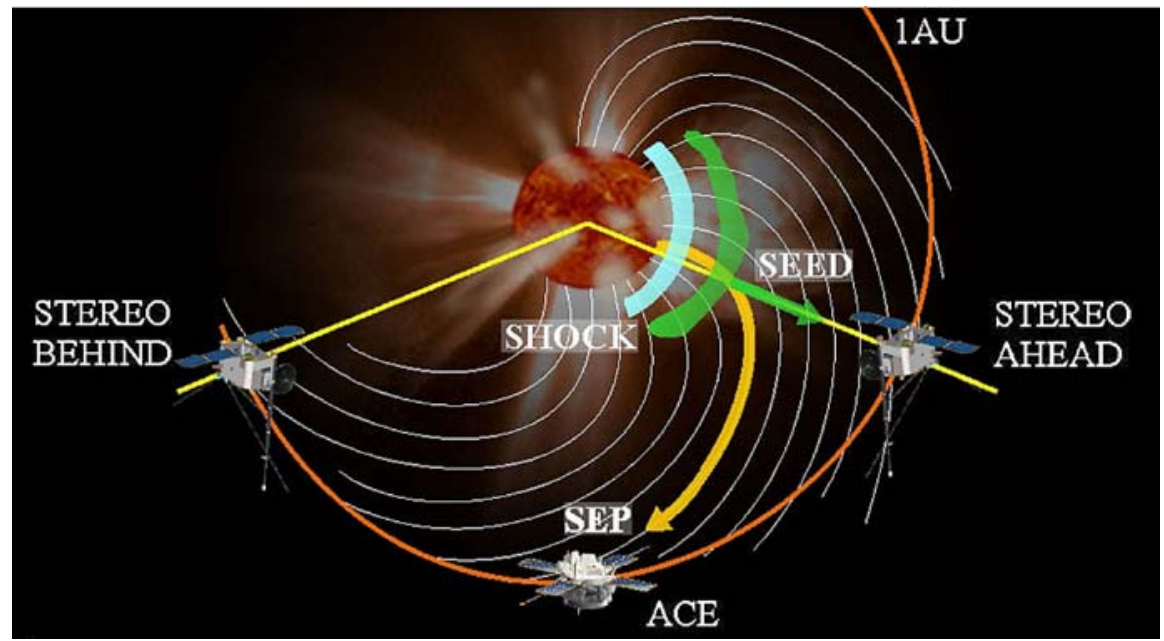

Fig. 3 Sketch of geometry of a western hemisphere shock-associated SEP event. The shock (light blue) accelerates particles out of the seed population (green) in its path. The energetic particles then follow (yellow arrow) the interplanetary magnetic lines that direct them east of the shock. For the geometry shown, STEREOAhead and ACE are required to observe both the seed population and the energetic particles; for other shock locations, different pairs of spacecraft would be used

larger than if the seeds were solar wind. In addition, the suprathermals show a much wider range of abundance variation than the solar wind; for example, the Fe/O ratio in SEPs varies by over a factor of $\sim 100$, while in the solar wind the variation is closer to a factor of $\sim 5$. These considerations will critically affect the abundances of the energized ions, as has been observed for traveling interplanetary shocks (Desai et al., 2003).

In order to explore the relationship between the energetic particle populations and the seed particles, it is important to observe the seed particles associated with energetic particle events. In the case of traveling interplanetary shocks this can be done in an approximate way by measuring suprathermals a day or two upstream of the shock-associated particle enhancements. When this is done, it is found that there is a significant correlation between the suprathermal ion composition and spectra, and the composition and spectra of the energetic particles (Desai et al., 2003). This is an indirect comparison since the upstream suprathermals are not the ones that encounter the shock. However the correlation is apparently preserved since the interplanetary shock is accelerating particles locally, and so the upstream particle properties are correlated, on average, with those accelerated by the shock.

For the case of large SEP events, this correlation no longer holds (Desai et al., 2006a), presumably because the acceleration site is closer to the Sun. Figure 3 sketches the geometry in the case of a western hemisphere shock-associated SEP event. The shock is most effective at accelerating particles close to the nose of the shock front (e.g., Reames, 1999). Once energized, the particles follow the interplanetary magnetic field spiral, and move east of the shock front as shown in Figure 3. Observations at a single point are unable to measure both the seed and energetic particle populations, but the STEREO spacecraft, supplemented by missions at L1 such as ACE and Wind will be able to make the required multi-point measurements. This is just one example of how the STEREO in-situ instruments will permit critical new measurements in studies of energetic particles in the interplanetary medium. 


\subsubsection{Large Solar Particle Events}

Large SEP events produce the highest energy particles of any mechanism in the solar system, and their ionizing radiation can disrupt or damage space systems. The shocks in many of the largest events are associated with Coronal Mass Ejections (CMEs) that can be imaged by the two STEREO spacecraft, opening the opportunity to explore the relationship between the $\mathrm{CME} /$ shock and energetic particle population. Figure 4 shows fluences of major ions accelerated in the 2003 November 2 event, which was one of several in the so-called "Halloween" series of SEP events (from Mewaldt et al., 2005). The energy spectra show "breaks" or roll-overs that occur at different energies for different species. The location of these spectral breaks may to be related to the details of the energetic particle escape from the accelerating shock (Ellison et al., 1985; Li et al., 2005), but it is also possible that diffusion effects in the interplanetary medium play the dominant role (Cohen et al., 2005). Measurements with one of the STEREO spacecraft can trace CMEs moving out from the Sun, while the other

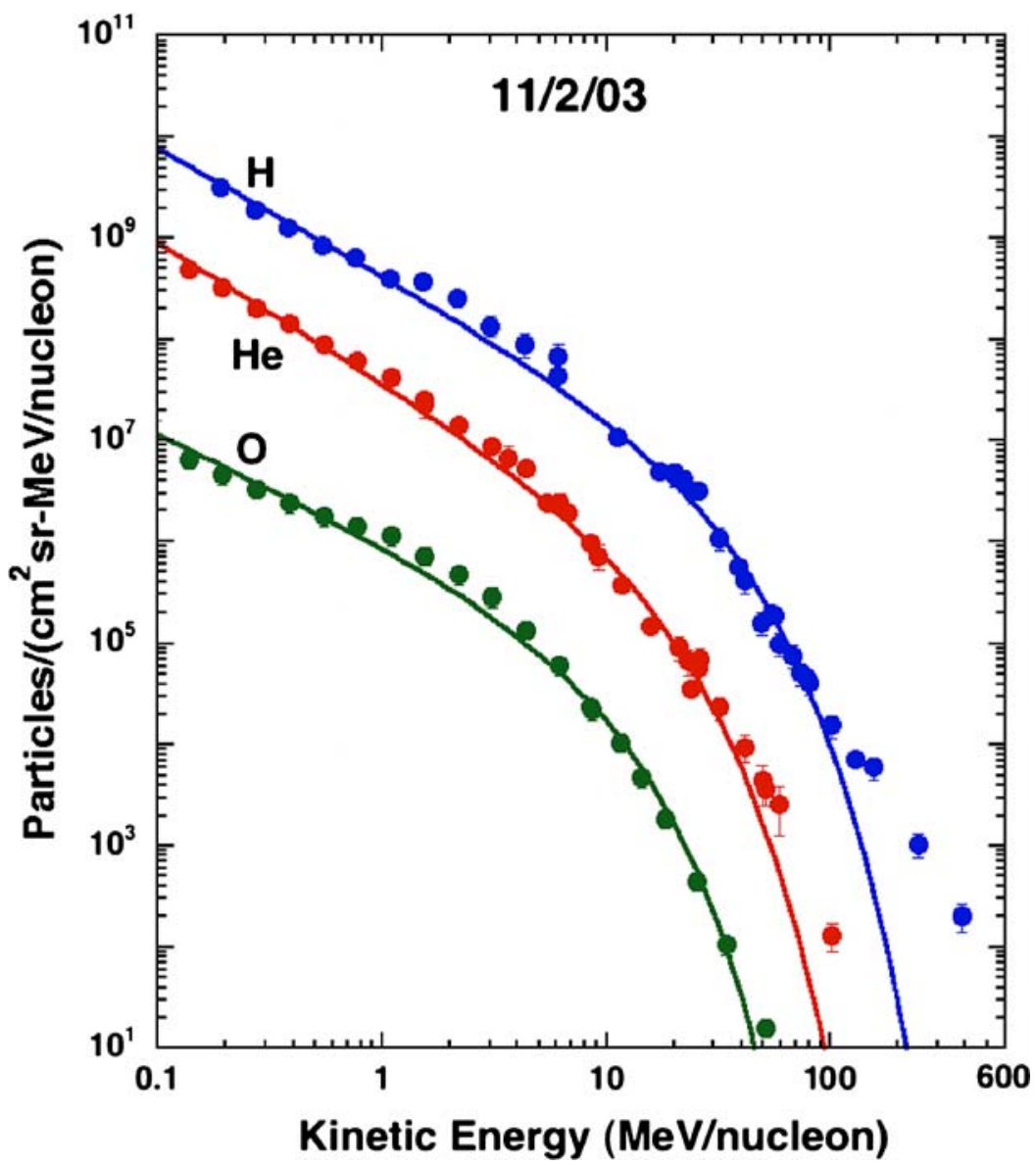

Fig. 4 Fluences of major energetic ion species in the 2003 Nov. 2 event show power law dependence at energies below a few $\mathrm{MeV} /$ nucleon, followed by steepening at higher energies. Notice that the spectra steepen at different kinetic energies for different species (from Mewaldt et al., 2005) 


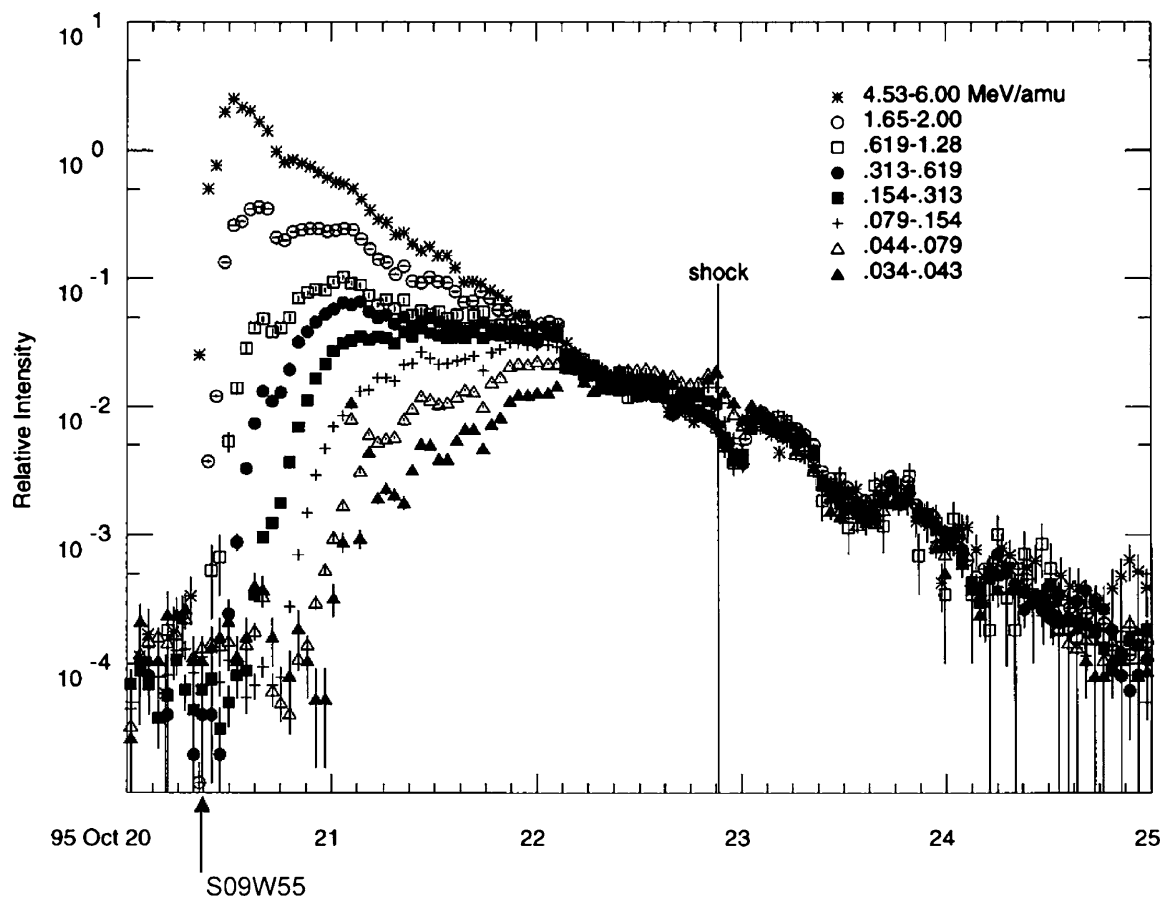

Fig. $5{ }^{4} \mathrm{He}$ intensity profiles for the 1995 October 20 event normalized to the decay phase intensities after October 22. Other species showed this same behavior (from Reames et al., 1997a). The parent event at the Sun was a flare of importance $0 \mathrm{~F}$ and X-ray intensity M1

spacecraft will simultaneously observe the energetic particles from the shock in front of the CME, thereby enabling critical studies of the relationship between particle energy spectral slopes and breaks and the initiating CME-driven shocks in large SEP events.

Although the event-integrated fluences shown in Figure 4 from low energies out to a few $\mathrm{MeV} /$ nucleon follow a single power law, there are dramatic differences in the time evolution of the particle intensities over this energy range. Figure 5 illustrates this for an event at S09W55 on 1995 October 20 (from Reames et al., 1997a). In this figure, the intensities have been adjusted to the same intensities during the decay phase starting on October 22. Even though the parent event in this case was magnetically well-connected to Earth, the intensity increases at low energies were very slow, with the 34-43 keV/nucleon channel beginning to increase almost 12 hours after the $4.53-6.00 \mathrm{MeV} /$ nucleon channel intensity had peaked. This event had been preceded by an interplanetary shock on October 18 and some of the slow rise at low energies may be due to turbulence associated with the earlier shock. In an event of this kind, however, with the low energy intensity peaks lagging the higher energy onset by 1-2 days, solar corotation may play an important role (Reames et al., 1997a) since at this late period the magnetic field lines are $>20^{\circ}$ from the shock center which had been sampled by the high energies when the event began. Since acceleration across the shock front is not uniform, corotation will significantly affect the low energy intensity profiles (Reames et al., 1997b). Behind the shock intensities are generally constant, and that may be responsible for the constant decay rates over the broad energy range shown in the figure (Reames et al., 1997b). However, alternate explanations are also possible (e.g., Sollitt et al., 
2003; Sollitt, 2004). For example if the SEPs are released from near the Sun and diffuse into a large scattering volume, the decay timescales will depend only on convection and adiabatic deceleration, which may be nearly constant over a significant energy range. This would result in energy and species independent decay time constants as seen in Figure 5. In cases where the scattering free-escape outer boundary is closer to the observer, then particle speed and mean free path can lead to decay time constants that vary with both energy and species (Sollitt et al., 2003; Sollitt, 2004), in contrast to the behavior shown in Figure 5. By probing the properties of these particle populations at widely varying longitudes, these contrasting models can be tested.

\subsubsection{Traveling Interplanetary Shocks}

In the large SEP event shown in Figure 5, the associated shock passage has virtually no effect on the high energy intensities. For the low energies, however, the situation is quite different; indeed, for the 34-43 keV/n O channel, the intensity for the whole event peaks at the shock passage. At energies above $\sim 10 \mathrm{MeV} /$ nucleon intensity peaks at shock passage are only occasionally observed, while at $\sim 1 \mathrm{MeV}$ /nucleon peaking at shock passage is observed frequently. As an example, Figure 6 shows low energy ion fluxes associated with an interplanetary shock whose parent activity was a halo CME originating near the solar central meridian at 23:23 on 2000 October 9 (http://cdaw.gsfc.nasa.gov/CME_list/UNIVERSAL/). The figure shows no promptly arriving particles for this event; however, the interplanetary intensities at low energies began to rise above background about 24 hours later, fully $\sim 2$ days before the shock arrived (see also Desai et al., 2001; Vandegriff et al., 2005). Assuming an average travel speed of $\sim 600 \mathrm{~km} / \mathrm{s}$, this event was detectable at 1 AU after the shock reached only $\sim 0.3$ AU. Coordinating this information with optical data from the SECCHI instruments, it will be possible on STEREO to correlate the optical information from well within $1 \mathrm{AU}$ with the particle population observed at $1 \mathrm{AU}$. In the case of this particular event, at $1 \mathrm{AU}$ energetic $\mathrm{O}$ ions were detected up to $\sim 1.5 \mathrm{MeV} /$ nucleon, a broad enough range to compute particle energy spectra that could be compared with model calculations. The geometry of the STEREO spacecraft (see Figure 3) will allow studies of this kind to be carried out for a very wide range of separations, with either of the STEREO spacecraft supplying the optical information, and the other the in-situ intensities.

The elemental composition and spectral shapes for shock-accelerated energetic particles carry information about the seed population and acceleration mechanism. Examining events where the peak intensities occur close to shock passage at 1 AU, Desai et al. $(2003,2004)$ characterized the average abundance and spectral properties of 72 interplanetary shock events. They found that the average abundances of the shock-accelerated particles had no simple relationship to solar wind abundances, but found instead that they were significantly correlated with the average abundances in the suprathermal ion population upstream of the shock. Indeed, it was found that in some cases the shock-accelerated particles had abundances of the rare isotope ${ }^{3} \mathrm{He}$ greatly in excess of solar wind values (Desai et al., 2001), which could only be due to acceleration of suprathermals from impulsive SEP events (see below). Figure 7 (left panel) shows spectra from the 1999 June 26 event which accelerated particles to several $\mathrm{MeV} /$ nucleon (Desai et al., 2004). The spectra are close to power laws at energies below $\sim 1 \mathrm{MeV} /$ nucleon, and then roll over at higher energies. The Fe spectrum is steeper than the $\mathrm{O}$ spectrum at all energies, causing a systematic decrease in the $\mathrm{Fe} / \mathrm{O}$ ratio as a function of energy (right panel). Desai et al. (2004) concluded that the shock acceleration mechanism 


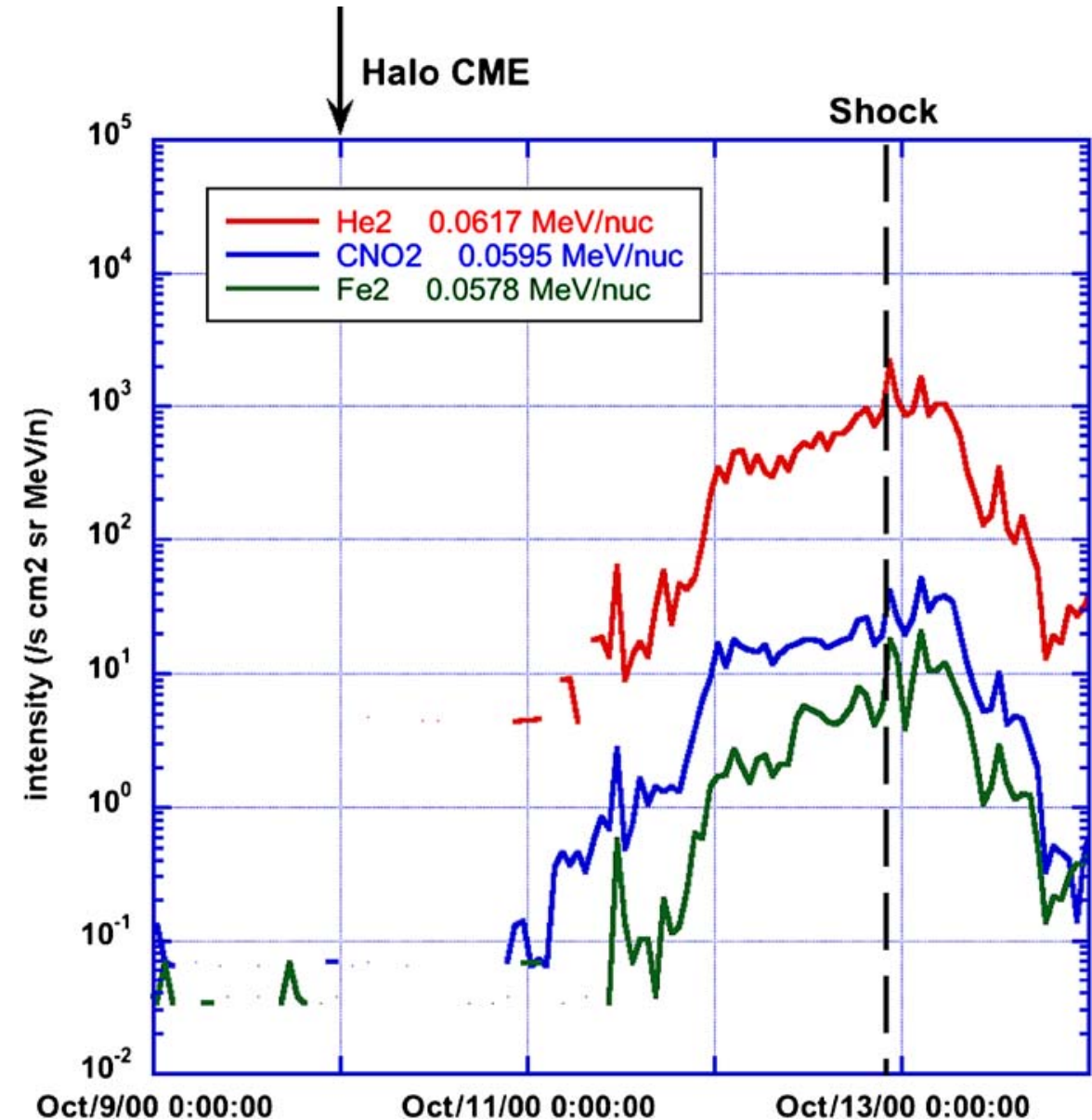

Fig. 6 Hourly average intensities of $\sim 60 \mathrm{keV} /$ nucleon $\mathrm{He}, \mathrm{C}+\mathrm{N}+\mathrm{O}$, and Fe from the Wind/STEP instrument for the 2000 October 13 shock. Notice that the interplanetary intensities begin to rise almost 2 days before the shock reaches 1 AU. On STEREO, an event of this kind would allow comparison of in-situ particle intensities with optical observations from SECCHI over an extended time interval

was less efficient at accelerating higher rigidity particles, and so Fe, with its higher $M / Q$ ratio than $\mathrm{O}$, was accelerated less efficiently.

\subsubsection{Impulsive Solar Flares}

In addition to large SEP events, there are "impulsive" SEPs, which are characterized by large enrichments of heavy ions and electrons, and enormous enrichments of the rare isotope ${ }^{3} \mathrm{He}$ as well as ions heavier than Fe (e.g. review by Reames, 1999, 2000; Mason et al., 2004). These events are infrequently associated with CMEs (Kahler et al., 2001). Recently Wang et al. (2006) and Pick et al. (2006) identified source regions for several impulsive events using energetic particle, optical, and imaging radio data. They found that in essentially every case, the event site was next to a coronal hole containing Earth-directed open field lines. The sites studied by Wang et al. (2006) were typically small, flaring active regions of the kind associated 

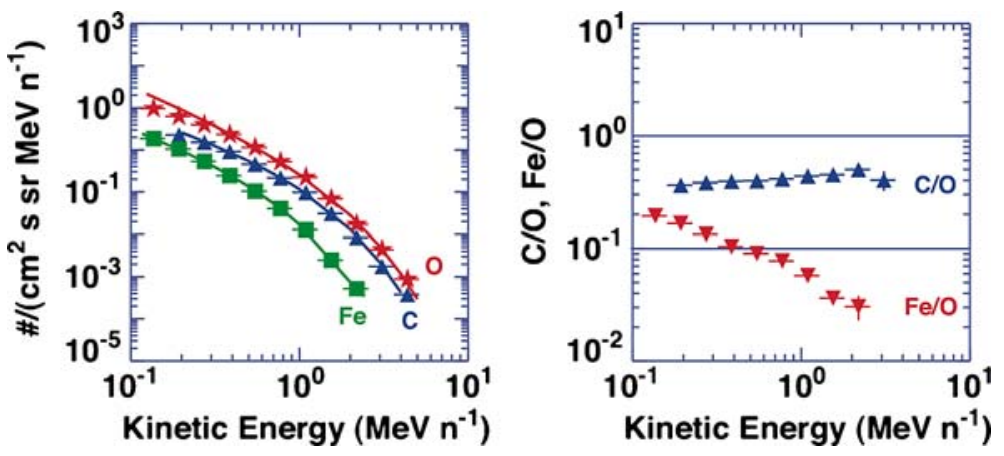

Fig. 7 Energy spectra (left panel) and abundance ratios (right panel) for the 1999 June 26 interplanetary shock event (event \#13 from Desai et al., 2004). The decrease of Fe/O with increasing energy is typical, and indicates that the acceleration mechanism is less efficient for higher rigidity ions

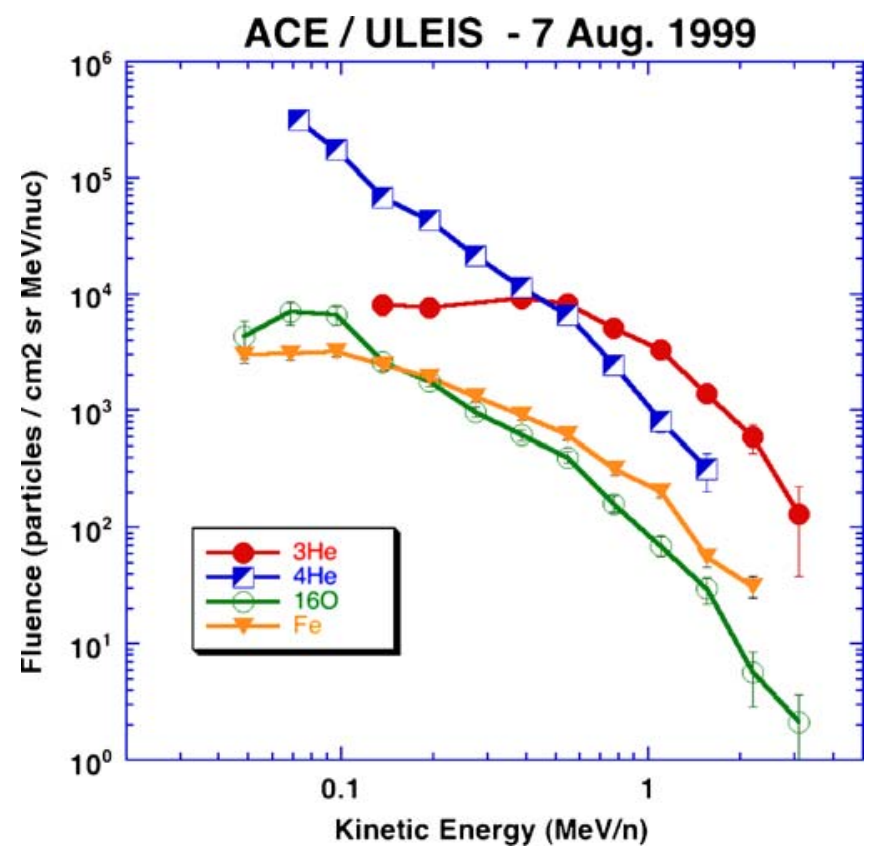

Fig. 8 Energy spectra for the 1999 August 7 impulsive SEP event; notice that the ${ }^{3} \mathrm{He}$ spectral shape is radically different from ${ }^{4} \mathrm{He}$, leading to a strong energy dependence of the ${ }^{3} \mathrm{He}:{ }^{4} \mathrm{He}$ ratio (from Mason et al., 2000)

with EUV jets. These jets have been studied extensively using Yohkoh observations, and have been interpreted as signatures of magnetic reconnection between open and closed magnetic field lines (e.g., Shimojo et al., 2000).

Figure 8 shows ion spectra from an impulsive ${ }^{3} \mathrm{He}$-rich event on 1999 August 7 (Mason et al., 2000). This event was associated with an M1.2 X-ray flare at N18W40. Notice that the energy spectra have strikingly different shapes, where the ${ }^{3} \mathrm{He}$ rolls over at lower energies, as does the Fe spectrum, while the ${ }^{4} \mathrm{He}$ spectrum is a power law. The ${ }^{3} \mathrm{He}:{ }^{4} \mathrm{He}$ ratio is a strong 
function of energy (see also Möbius et al., 1982). Different spectral forms such as shown in Figure 8 are commonly observed at lower energies; however, some of these events show power-law, or broken power-law spectra as well, with most all the species having similar spectra (Mason et al., 2000; Mason et al., 2002). These different spectral properties indicate that there may be more than one acceleration process at work in impulsive flares (Mason et al., 2002).

There are only a handful of impulsive SEP events that have been observed on multiple spacecraft (e.g., Mason et al., 1989) and these were with instruments with much higher energy thresholds than the STEREO instruments. Thus, STEREO will allow the first systematic studies of impulsive events that probe the longitude dependence on a case-by-case basis. Impulsive events come from rather compact regions and are observed over at most a few $10 \mathrm{~s}$ of degrees of longitude. In addition, "drop-outs" observed in impulsive event intensities can be best understood if the source region is small (Mazur et al., 2000). Particle injection timing studies for these events show that if the interplanetary transport is scatter-free, then the ions appear to be injected roughly an hour later than the low energy electrons (Wang et al., 2005). If the ion injection is significantly later than the electrons, this has important implications for the acceleration process or site. STEREO will allow studies of multiple impulsive events whose magnetic connection to the spacecraft will be somewhat different in each case, making it possible to probe the role of scattering versus acceleration in the interpretation of the data at 1 AU.

Due to the common frequency of occurrence of impulsive events during active periods, impulsive event material is present in the interplanetary medium for a substantial fraction of the time (e.g., Wiedenbeck et al., 2003). This material can be accelerated by shocks associated with large solar energetic particle events and detected due to the enrichment of ${ }^{3}$ He sometimes seen in Large Solar Energetic Particle (LSEP) events (Mason et al., 1999a). Thus, in interpreting shock-associated acceleration in LSEP events, it is essential to be able to monitor suprathermal particle intensities for the presence of impulsive SEP material, since this material is an important component of the seed particle pool.

\subsubsection{Corotating Interaction Regions (CIRs)}

Corotating interaction regions (CIRs) are regions of interplanetary space where fast- and slowsolar wind streams interact to form forward and reverse shocks that can accelerate particles to multi-MeV energies (Richardson et al., 1984; Richardson, 1985; review by Mason et al., 1999b). The particle intensities in these events often peak well outside $1 \mathrm{AU}$, however, in many cases the particles are observed at $1 \mathrm{AU}$ as shown in Figure 9. Since CIRs such as the one shown are in nearly a steady state lasting for multiple solar rotations, the width of the event is a measure of its angular extent ( $\sim 4$ days in this case, or $\sim 50^{\circ}$ of longitude). By measuring CIRs using the two STEREO spacecraft, it will be possible to test the extent to which profiles such as that in the figure are actually unchanging over short periods. In addition, CIRs are important contributors to the interplanetary suprathermal ion pool, since they can last for many solar rotations and reach fairly high intensities for low energy ions. This is shown in Figure 10, which plots the suprathermal $\mathrm{Fe} / \mathrm{O}$ and $\mathrm{C} / \mathrm{O}$ ratios over the past solar cycle (Desai et al., 2006b). The figure shows that during the declining phase of the prior cycle (1994-96) the suprathermal ion pool measured during "quiet" days had high C/O and low Fe/O ratios typical of CIRs. As solar activity increased, these ratios systematically changed over to values much closer to SEPs, namely lower $\mathrm{C} / \mathrm{O}$ and relatively high $\mathrm{Fe} / \mathrm{O}$ ratios. Since the activity maximum in 1999-2002, they are now returning to the values closer to the prior solar minimum values. Since this suprathermal ion pool is the source of energetic particles accelerated by CME- 


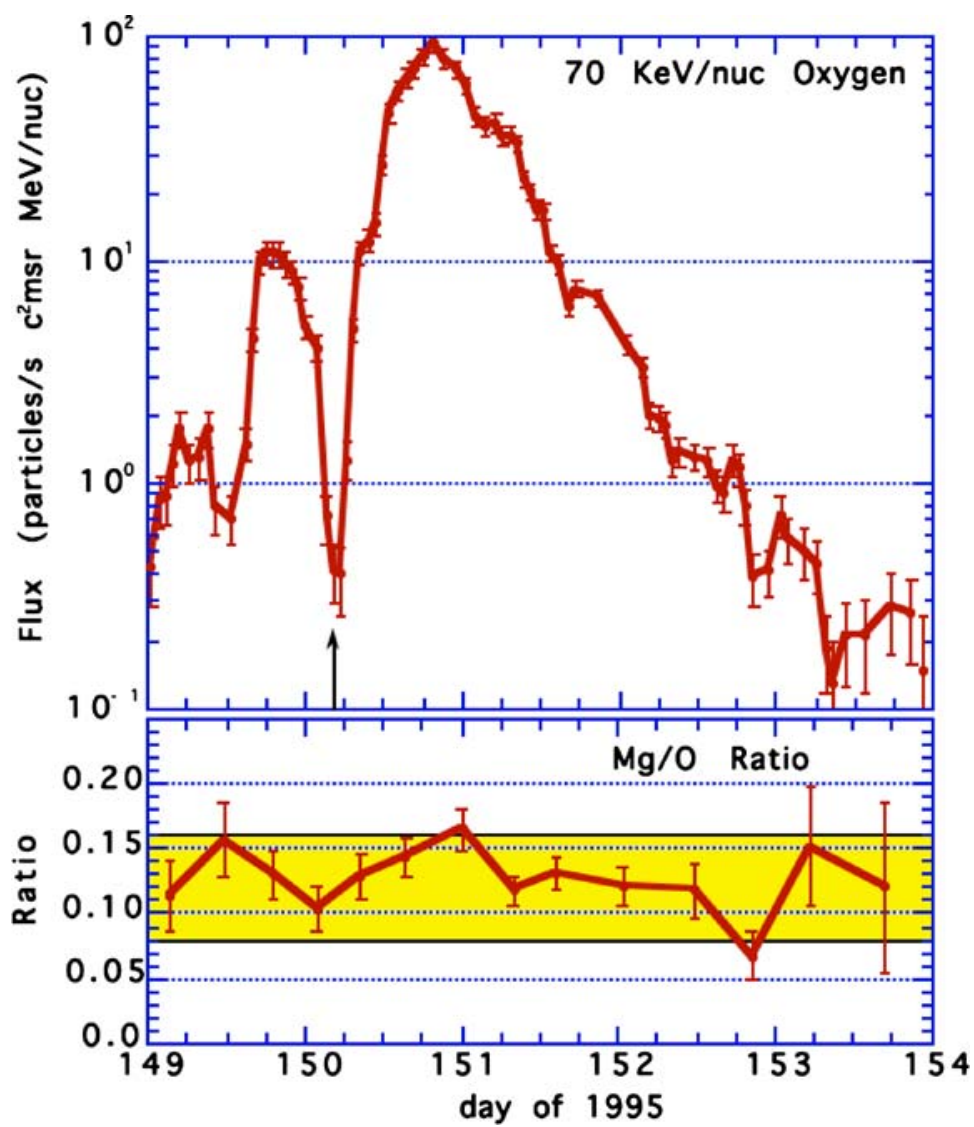

Fig. 9 Top: Intensity of $70 \mathrm{keV} /$ nucleon O ions observed by the Wind/STEP instrument during a CIR in 1995. Arrow marks the strong decrease in intensity associated with the passage of the stream interface. Bottom: ratio of $\mathrm{Mg} / \mathrm{O}$ during the $\mathrm{CIR}$, compared with solar wind $\mathrm{Mg} / \mathrm{O}$ value in slow- and fast-solar wind streams (yellow shaded box) (Mason et al., 1997)

driven interplanetary shocks, understanding its composition and temporal variations is key for many STEREO observations. Multiple spacecraft observations will make it possible to put together a much more complete picture than is available now.

\section{Design Requirements}

The preceding discussion illustrates why measurements of energetic particles on each of the STEREO spacecraft are required to address one of the key mission objectives, namely to discover the mechanisms and sites of energetic particle acceleration in the low corona and interplanetary medium. This requires knowledge of the particle energy spectra, directionality, and intensity, as well as the timing of these quantities compared with other observables at the Sun and in the interplanetary medium.

The Suprathermal Ion Telescope (SIT) for the STEREO mission is one of a coordinated group of sensors designed to give comprehensive coverage of ions and electrons from the energy range just above the solar wind to low energy cosmic rays (see Luhmann et al., 2007). 


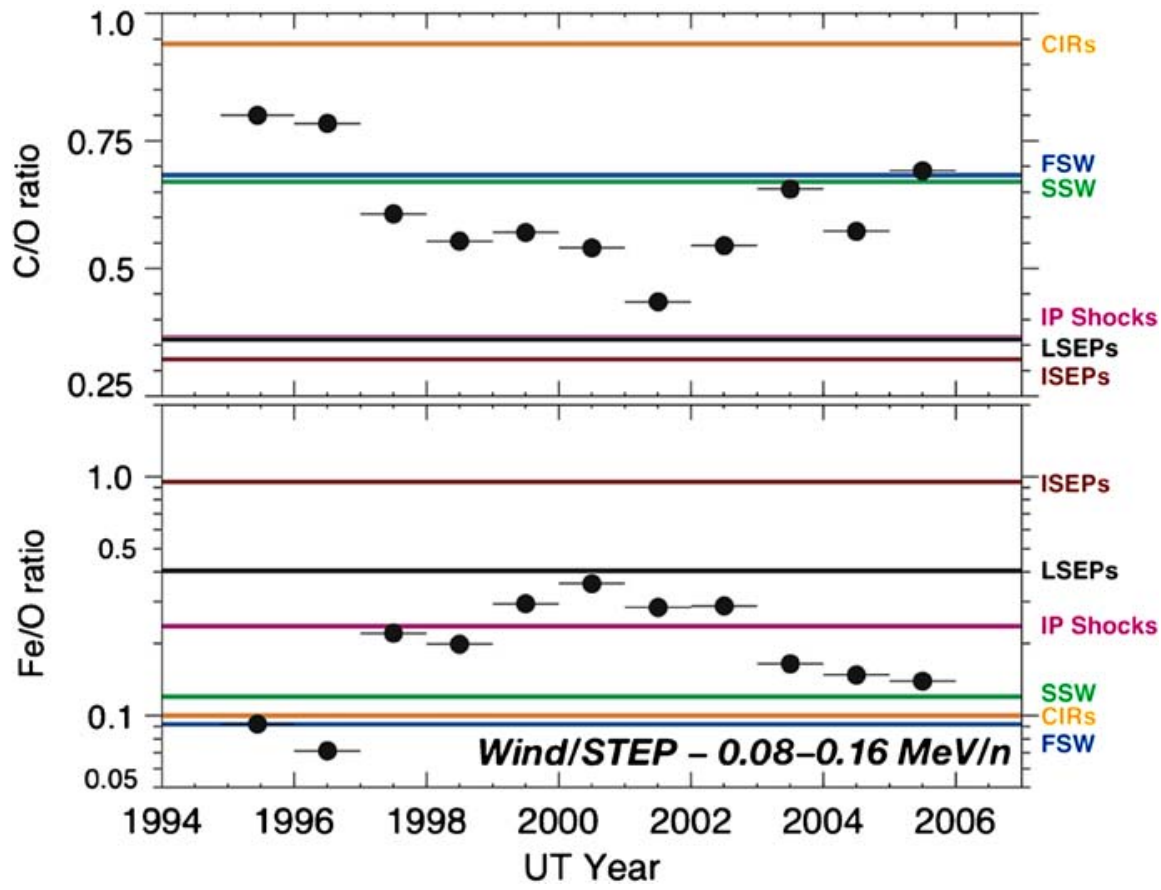

Fig. 10 80-160 keV nucleon ${ }^{-1} \mathrm{C} / \mathrm{O}$ (upper panel) and $\mathrm{Fe} / \mathrm{O}$ (lower panel) ratios obtained by Wind/STEP during the quiet-days of each year over a complete solar cycle. Horizontal lines represent average abundances measured in various heliospheric ion populations. Orange: corotating interaction regions (CIRs, Mason et al., 1997); brown: ${ }^{3} \mathrm{He}$-rich or impulsive solar energetic particle events (ISEPs: Mason et al., 2002); black: gradual or large solar energetic particle events (LSEPs: Desai et al., 2006a); purple: CME-driven IP shock events (IP shocks: Desai et al., 2003); blue: fast solar wind (FSW); and green: slow solar wind (SSW). Average solar wind values are taken from von Steiger et al. (2000)

In addition to SIT, IMPACT includes other sensors optimized to cover the required energy range: the Solar Electron and Proton Telescope (SEPT, Müller-Mellin et al., 2007), the Low Energy Telescope (LET, Mewaldt et al., 2007), and the High Energy Telescope (HET, von Rosenvinge et al., 2007). As shown in Figure 1, this energy range covers a broad range of phenomena central to the studies on STEREO. However, in 1997 when the STEREO science definition team (SDT) defined a model payload for STEREO, the critical role of the suprathermal ion population had not yet been realized since most of the discoveries in this area have come from the ACE mission that was launched only $\sim 4$ months before the final SDT report. The STEREO model payload therefore made no allocation to cover suprathermal heavy ions. By the time the Announcement of Opportunity for STEREO was released in mid1999 the role of the suprathermals was becoming increasingly clear to the science community, and the IMPACT science team proposed covering this additional energy range even though it was not in the model payload. In order to keep the cost and spacecraft resource impacts at a minimum, it was decided to use a legacy design, namely the dual-telescope Suprathermal through Energetic Particle (STEP) detector which is part of the EPACT investigation on the Wind mission (von Rosenvinge et al., 1995). While it would have been desirable to increase the solid angle coverage to accommodate the fact that STEREO is a stabilized spacecraft (while Wind is a spinner), resource limitations ruled this out and the team proposed a single SIT sensor for each spacecraft. 
Table 1 SIT design goals

\begin{tabular}{|c|c|c|}
\hline & Goal & Science driver \\
\hline Geometrical factor & $0.29 \mathrm{~cm}^{2} \mathrm{sr}$ & $\begin{array}{l}\text { Adequate counting statistics for } \\
\text { - weak shock events } \\
\text { - impulsive SEPs }\end{array}$ \\
\hline Particle species range & $2 \leq Z \leq 28$ & $\begin{array}{l}\text { - He-Ni covers most abundant material } \\
\text { above protons (protons covered by SEPT) } \\
\text { - need to distinguish particle populations } \\
\text { from: } \\
\text { - gradual SEP events } \\
\text { - impulsive SEP events } \\
\text { - corotating interaction regions }\end{array}$ \\
\hline Mass resolution & $\sigma_{m} / m=0.1$ & - resolve ${ }^{3} \mathrm{He}$ from ${ }^{4} \mathrm{He}, \mathrm{C}$ from $\mathrm{O}$ \\
\hline & $1 /$ day $<R<10^{5} / \mathrm{s}$ & $\begin{array}{l}\text { - Low background to allow study of } \\
\text { impulsive SEPs } \\
\text { - Upper limit allows coverage of large SEP } \\
\text { events }\end{array}$ \\
\hline
\end{tabular}

The overall design goals for SIT are summarized in Table 1. By meeting these goals, SIT will cover the energy range from the suprathermals above the solar wind, up to the energy threshold of the LET sensor (Mewaldt et al., 2007). It will also be able to resolve key species that permit discrimination between the gradual SEP, impulsive SEP, CIR, and other energetic particle populations. Finally, the goals require both low background for small event periods, and high analysis rates for large SEP events so that the particle distribution functions can be measured under all conditions.

\section{Instrument Description}

\subsection{Telescope Cross Section}

Figure 11 shows cross sectional views of the SIT telescope, which is a time-of-flight mass spectrometer. Ions enter the telescope chamber through thin Ni foils, and strike a Si solidstate detector mounted a distance $L=10 \mathrm{~cm}$ behind the foil. When the ions exit the inner $\mathrm{Ni}$ foil, secondary electrons are emitted and these are accelerated to $\sim 1 \mathrm{kV}$ and directed to a chevron-microchannel plate (MCP) pair that provides a fast "start" pulse for the instrument electronics. If the ions pass through the surface of the Si detector, secondary electrons are also emitted, and these are accelerated and directed to another set of MCPs that provide a fast "stop" signal so that the time-of-flight $\tau$ is obtained. The electrostatic acceleration and focusing is done using techniques used in vacuum-tube technology (Harman, 1953) and avoids the use of accelerating grids. The particle is stopped in the solid state detector, where its residual energy $E_{\mathrm{SSD}}$ is measured. The mass of the ion is obtained from:

$$
m=2 E_{\mathrm{SSD}}\left(\frac{\tau}{L}\right)^{2}
$$

The mass identification in Equation (1) is approximate due to the non-linear response of the solid state detector for very low energy ions (e.g., Ipavich et al., 1978; Galvin, 1982). However, since the main element masses are known this is not a problem since all that is required 


\section{Suprathermal Ion Telescope (SIT)}

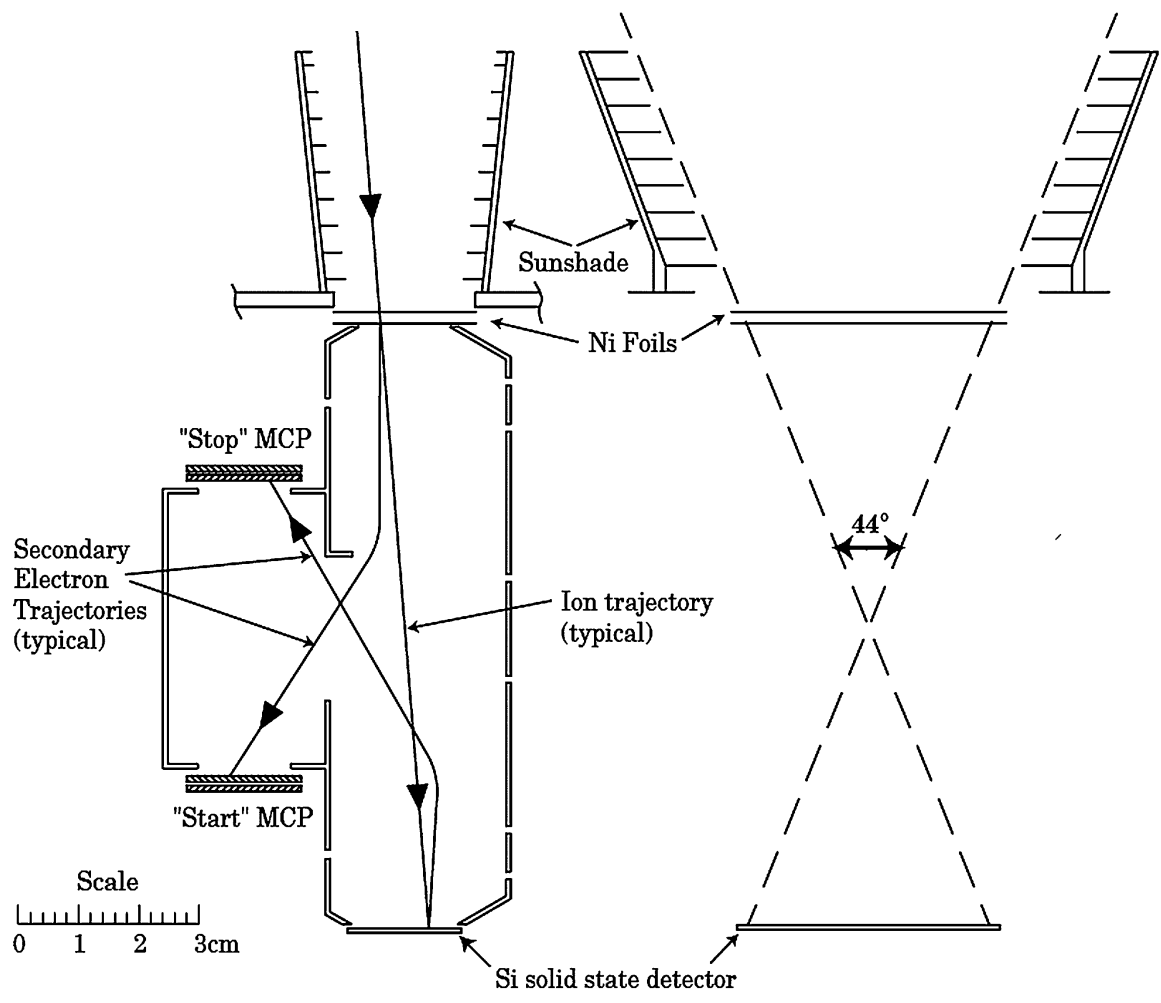

Side View

Top View

Fig. 11 Schematic cross sections of the Suprathermal Ion Telescope (SIT, see also von Rosenvinge et al., 1995). Acceptance angle for left view is $17^{\circ}$. See text for a description of the operating principles

for identification is a separation of different mass ions. The incident energy is determined from the time-of-flight measurement after taking account of the foil thickness and non-linear response of the solid state detector at low energies. At energies below $\sim 100 \mathrm{keV} /$ nucleon, nonionizing collisions in the solid-state detector limit the instrument resolution (Ipavich et al., 1978; Galvin, 1982). At energies above $\sim 1 \mathrm{MeV} /$ nucleon, dispersion in the time-of-flight measurement dominates the instrument resolution (for a more complete discussion see Mason et al., 1998). In the range $\sim 0.1-1.0 \mathrm{MeV} /$ nucleon, the mass resolution is $\sim \sigma_{m} / m=0.1$. A summary of detector properties and instrument response and resources is given in Table 2.

In order to keep the solid state detector temperature down, and to prevent solar UV from striking the front foil, there is a sunshade that extends for $\sim 3 \mathrm{~cm}$ outside the foils and limits the solid angle seen by the front foil. A closable cover (not shown in Figure 11) protects the foils during launch and is opened on orbit. Figure 12 is a photograph of the STEREO/Behind SIT sensor with door open during a pre-launch test. The sunshade is painted black, and the outer foil, mounted on an Au-coated grid, is clearly visible at the back. The cylindrical housing on the bottom of the sunshade contains a TiNi pin-puller that is fired by a spacecraft 
Table 2 SIT summary

\begin{tabular}{|c|c|}
\hline Item & Technique or details \\
\hline Objective & $\begin{array}{l}\text { Elemental and isotopic composition of interplanetary suprathermal ions from } \\
\text { CME-associated solar energetic particle events; impulsive }{ }^{3} \mathrm{He} \text {-rich solar flares, } \\
\text { interplanetary shocks, and Corotating Interaction Regions }\end{array}$ \\
\hline Technique & $\begin{array}{l}\text { Time-of-flight vs. residual energy using microchannel plate detection of secondary } \\
\text { electrons and solid state detector; } 10 \mathrm{~cm} \text { ion flight path }\end{array}$ \\
\hline Detectors & $\begin{array}{l}\text { Window foils: two } 40.7 \times 15.5 \mathrm{~mm} 1000 \AA \mathrm{Ni} \text { (each) mounted on } 90 \% \text { transparent } \\
\text { Au-plated BeCu etched mesh; supplied by LeBow Co., Goleta, CA. } \\
\text { Silicon ion implant detector: } 15 \times 40 \mathrm{~mm} \text { active area; thickness } 500 \mu \text {; active } \\
\text { surface metalization } 0.1 \mu \mathrm{Al} \text {, junction dead layer } 0.35 \pm 0.03 \mu \text {; noise pulser } \\
\text { width } 25 \mathrm{keV} \text { typical; supplied by Micron Semiconductor Ltd., Lancing, } \\
\text { Sussex, England } \\
\text { Microchannel plates: chevron, } 15 \times 37 \mathrm{~mm} \text { active area; thickness } 1.0 \mathrm{~mm} \text { each; } 25 \\
\text { micron pore size; bias angle } 8^{\circ} \text {; supplied by Galileo Electro-Optics Corp., } \\
\text { Sturbridge, MA }\end{array}$ \\
\hline Sensor & One on each STEREO spacecraft \\
\hline Electronics & $\begin{array}{l}\text { Solid state detector energy measurement: } \\
\text { 11-bit precision; threshold } 240 \mathrm{keV} \text {; high gain ramp maximum energy } \\
\sim 21 \mathrm{MeV} \text {; low gain ramp maximum energy } 174 \mathrm{MeV} \\
\text { Time of flight measurement: } \\
\text { 9-bit precision; range } \sim 2.5-125 \mathrm{~ns}\end{array}$ \\
\hline Geometry & $\begin{array}{l}\text { A } \Omega \text { : } 0.29 \mathrm{~cm}^{2} \text {-sr each unit after correction for foil mesh transparency } \\
\text { FOV: } 44^{\circ} \times 17^{\circ} \text { field of view; } 44^{\circ} \text { angle lies in ecliptic plane; } 17^{\circ} \text { angle is } \\
\text { perpendicular to ecliptic plane; center line of } 44^{\circ} \text { cone is } 60^{\circ} \text { west of S/C-Sun } \\
\text { line }\end{array}$ \\
\hline Charge Interval & $2 \leq Z \leq 26$ \\
\hline \multirow{2}{*}{$\begin{array}{l}\text { Energy Intervals for } \\
\text { typical elements }\end{array}$} & He $123 \mathrm{keV} /$ nucleon $-8.1 \mathrm{MeV} / \mathrm{n}$ \\
\hline & $\begin{array}{l}\mathrm{C} 68 \mathrm{keV} / \text { nucleon }-8.1 \mathrm{MeV} / \mathrm{n} \\
\mathrm{O} 57 \mathrm{keV} / \text { nucleon }-8.1 \mathrm{MeV} / \mathrm{n} \\
\text { Fe } 28 \mathrm{keV} / \text { nucleon }-3.2 \mathrm{MeV} / \mathrm{n}\end{array}$ \\
\hline $\begin{array}{l}\text { Energy interval for } \\
\text { resolving }{ }^{3} \mathrm{He}\end{array}$ & $300-800 \mathrm{keV} /$ nucleon \\
\hline Mass resolution & $\sigma_{m} / m=0.1(\sim 0.1-1.0 \mathrm{MeV} /$ nucleon $)$ \\
\hline \multicolumn{2}{|l|}{ Resources } \\
\hline Mass & $1.63 \mathrm{~kg}$ (including thermal coatings and heaters) \\
\hline Power & 1.65 W (regulated; includes HVPS; solid state detector bias not included) \\
\hline Bit rate & $424 \mathrm{bits} / \mathrm{s}$ \\
\hline
\end{tabular}

signal on orbit to release the spring-operated door (top). A small shade on the left of the sunshade is for additional UV protection. The interior of the sunshade door is coated for thermal control. On the spacecraft, the entire assembly is covered with thermal blankets, and only the telescope opening is visible.

Figure 13 shows the SIT field-of-view (FOV) orientation. The narrower ( $17^{\circ}$ full width) FOV is bisected by the ecliptic plane. The larger ( $44^{\circ}$ full width) FOV is oriented so that its center line is $60^{\circ}$ from the spacecraft-Sun line. With this angle of orientation, the first foil is always shadowed by the sunshade. The other obvious constraint on the FOV is that it should look close to the average Parker magnetic field spiral line, a requirement that is met by this geometry. 


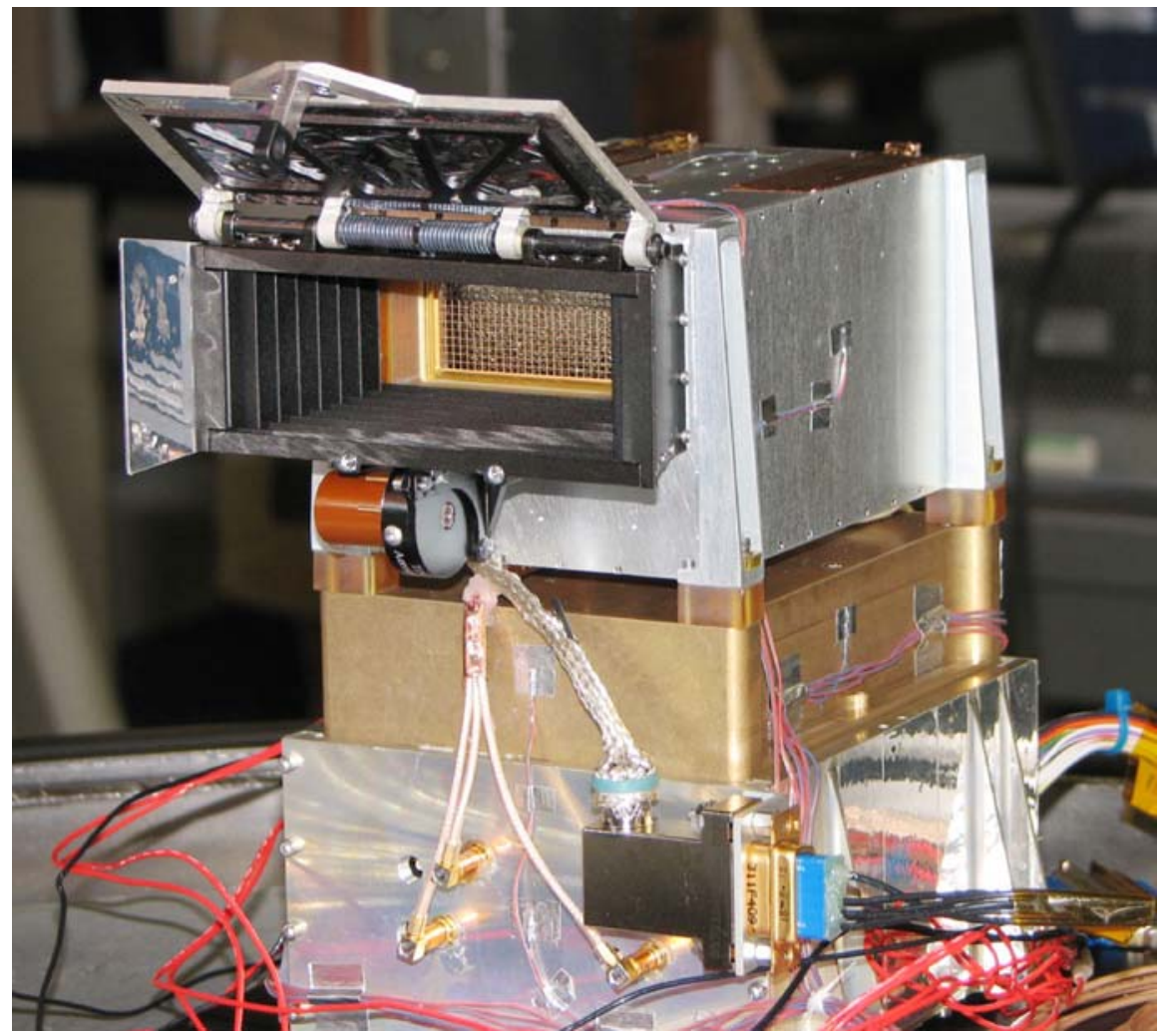

Fig. 12 SIT instrument for the STEREO Behind spacecraft shown in a thermal vacuum chamber after a test of the openable door. The telescope box is Al-colored; underneath it lies the gold-colored high voltage power supply for microchannel plate bias; beneath that is the electronics box that has reflective thermal coatings

\subsection{Functional Block Diagrams}

\subsubsection{Telescope Electronics}

Although the SIT telescope mechanical design is essentially identical to the Wind/STEP design, the electronics are a new design in order to take advantage of advances of the past $\sim 15$ years, and to meet the more stringent quality assurance requirements of the STEREO mission. Figure 14 is a functional block diagram of the SIT instrument. Dashed lines in the figure separate the three components of the instrument, namely the telescope box, high voltage power supply (HVPS) box, and the electronics box (see Figure 12). The HVPS supplies bias to the telescope MCP stacks, and to the sections of the housing that set up the electrostatic field that accelerates and deflects the secondary electrons to the MCPs. The initial operating $\mathrm{HV}$ setting of about $3100 \mathrm{~V}$ provides $900 \mathrm{~V}$ for the secondary electron assembly acceleration and deflection bias, and $930 \mathrm{~V}$ bias across each MCP and some additional voltage steps for interplate bias and bias of the collector plate. The design includes provision for raising the $\mathrm{HV}$ to compensate for loss of MCP gain; the maximum voltage is about $25 \%$ higher than the initial operating voltage, and can be increased in steps of $0.6 \%$ each. 


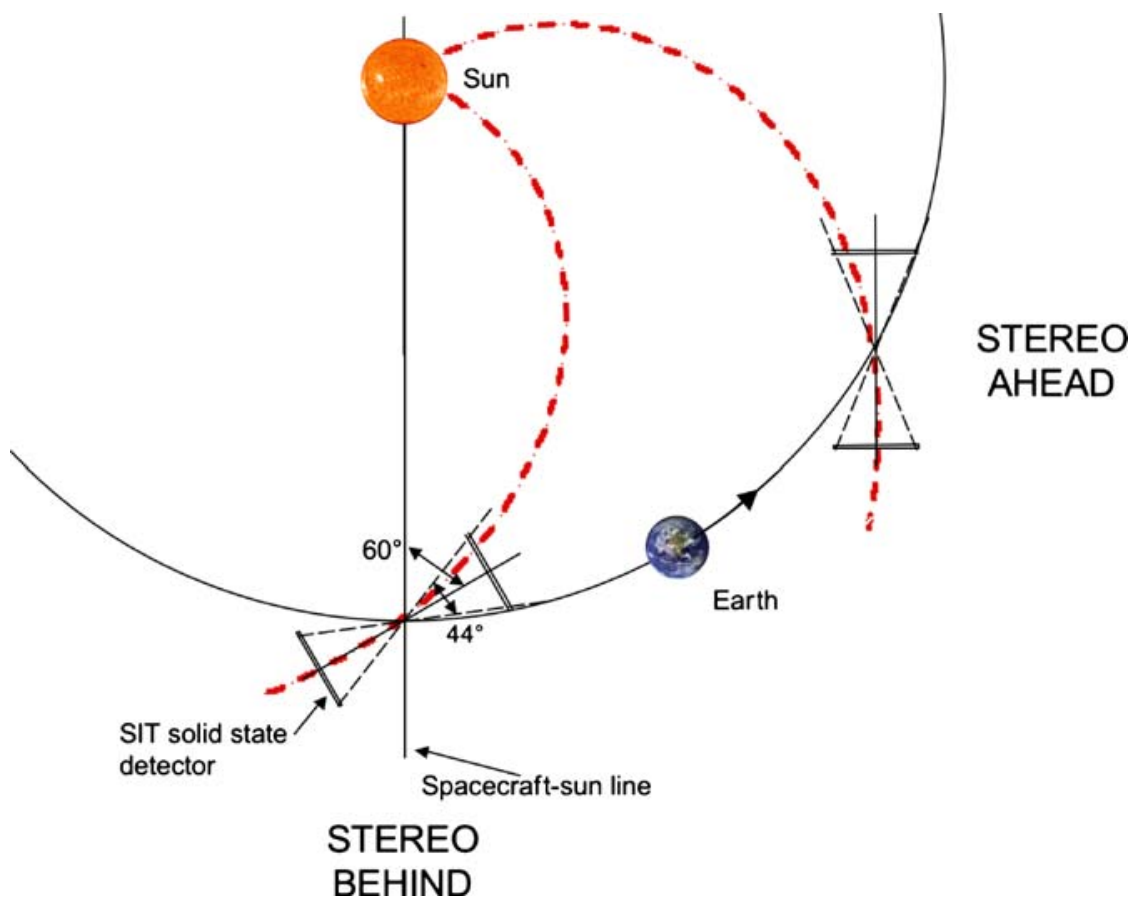

Fig. 13 Orientation of the SIT telescopes with respect to the spacecraft-Sun line. Dashed red spirals: typical interplanetary spiral magnetic field lines. The $44^{\circ}$ FOV lies in the ecliptic as shown, and the $17^{\circ}$ FOV is bisected by the ecliptic. The mounting of the SIT instruments on the AHEAD and BEHIND spacecraft is arranged as to achieve the same IMF look direction for both. Black arrow shows direction of Earth's orbital motion

Signals from the START MCP, STOP MCP, and solid state detector are processed in the electronics box. The Analog time-of-flight (TOF) board receives the telescope START and STOP MCP signals that go first to fast amplifiers followed by constant fraction discriminators (CFDs) that remove TOF "walk" arising from different signal amplitudes on the input. The electronics box houses four separate boards that are connected by a backplane motherboard. The CFD output signals are then sent to the Digital TOF board (DTOF, described below).

Solid state detector signals are sent to the Energy board, where they are amplified using Amptek hybrids. Every signal from the solid-state detector is split and then analyzed by both a high- and low-gain amplifier. Both the high- and low-gain signals are digitized and sent to the Logic Board. In addition, a discriminator output from the Si detector is sent to the DTOF for use in forming the event coincidence.

The digital time-of-flight system consists of two main components, a Time-to-Digital Converter (TDC) implemented in an ASIC, and an ACTEL FPGA that contains the necessary support circuitry. The power consumption is small ( $120 \mathrm{~mW}$ at $30 \mathrm{k}$ events/s). Figure 15 shows a functional block diagram of the DTOF board. The CFD START and STOP outputs from the ATOF board are split and sent to the TDC where they are digitized, and also to the DTOF board ACTEL for processing. The output TOF and input control signals are sent on serial lines to minimize connector size, and other signals from the Logic Board are required to check for coincidence between the START-STOP and solid state detector. The time range of the 9-bit TDC output is $0-128 \mathrm{~ns}$. 


\section{Suprathermal Ion Telescope (SIT) block diagram}

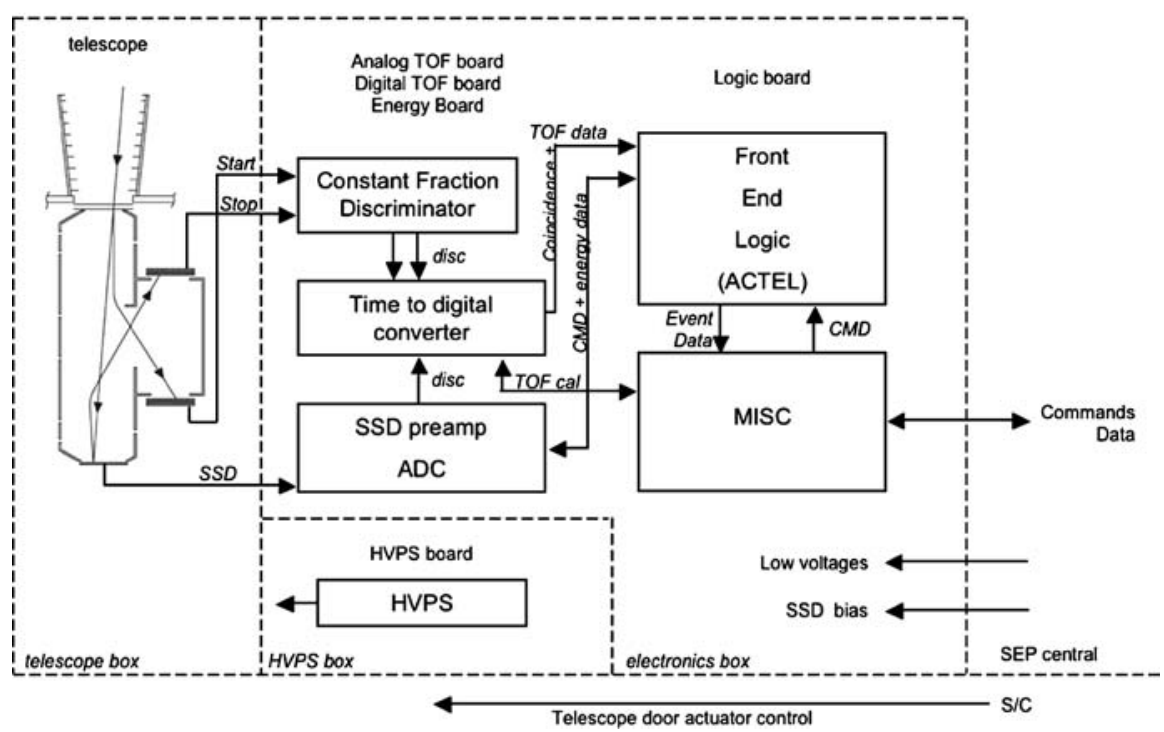

Fig. 14 Functional block diagram of the SIT instrument

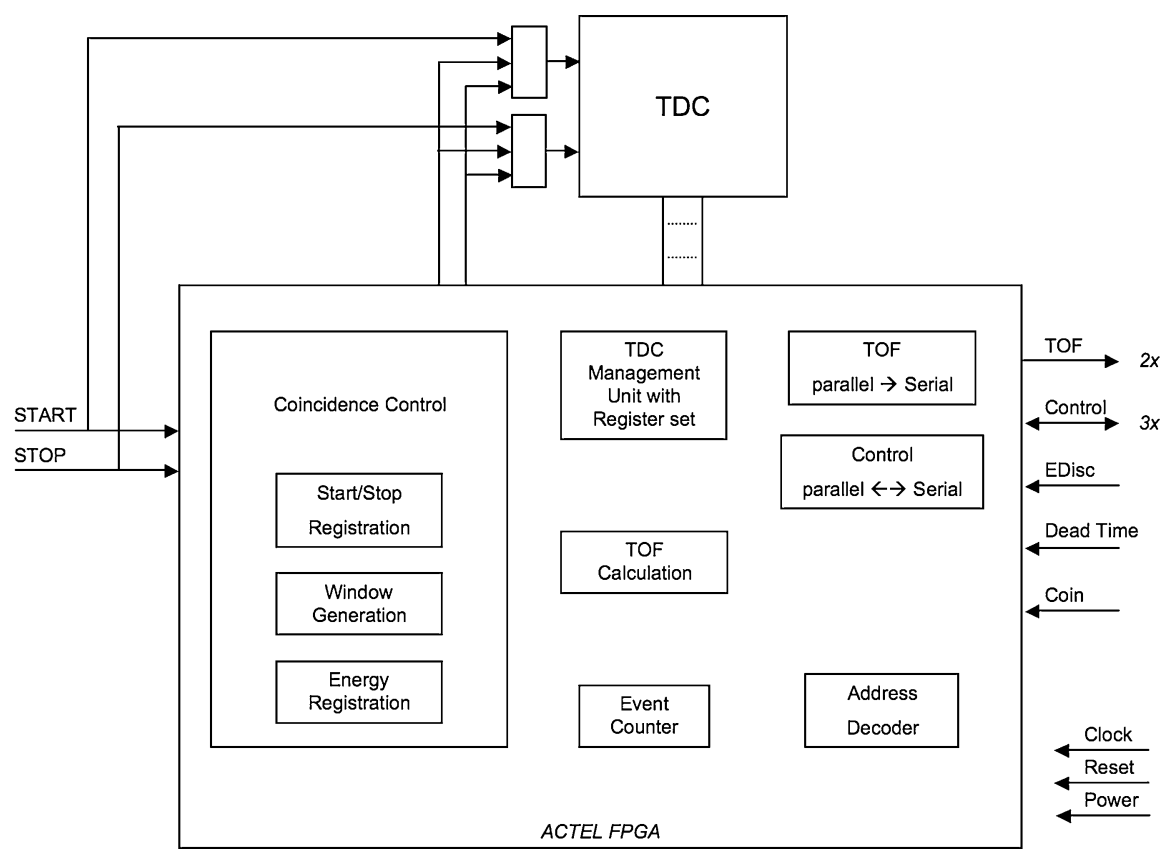

Fig. 15 Functional block diagram of the Digital Time-Of-Flight (DTOF) system 
The TOF system calibration is affected by temperature and voltage. It is therefore calibrated once per second by stimulating the TDC at a known fixed interval, and processing the resulting values. The Logic Board initiates the calibration, reads output values and does a short calculation whose results for gain and offset are written into the DTOF system, thus keeping the TOF system calibrated. The TOF gain and offset values are also telemetered in the instrument housekeeping. The TOF system also monitors the occurrence of multiple START or STOP signals for a given event, and sets flags for these conditions for each event. The system also provides count rates for START, STOP, and valid TOF events. The maximum event rate of the system is $>200 \mathrm{kHz}$, a factor of $\sim 50$ higher than the maximum anticipated event rate. Rate accumulators in the DTOF system are 16-bit counters that read to the MISC once per second where they are accumulated as 24-bit numbers. Rates with the potential to overflow the accumulator (i.e., $>65 \mathrm{kHz}$ ) are prescaled (see Table 3 ).

Digitized TOF and solid state detector signals along with timing and coincidence information are sent to the Logic Board, which processes each event. The front-end logic is contained in an ACTEL FPGA, that also contains the Minimum Instruction Set Computer (MISC, Mewaldt et al., 2007). SIT command states are also sent to the Logic Board ACTEL where they control event processing (see below). The output digital event data and count rate data is collected into telemetry packets in the MISC, then sent to the SEP Central Data Processing Unit (see Mewaldt et al., 2007) where it is time tagged. Housekeeping data is digitized in the Logic Board and sent to SEP Central for inclusion in a telemetry packet shared by other sensors in the IMPACT/SEP package. SEP Central also provides SIT with command data, low voltage power, and solid state detector bias.

\subsection{Data Outputs}

\subsubsection{PHA Data}

Each ion that triggers a START followed by a single STOP signal within $128 \mathrm{~ns}$ is a "VALID STOP" event and is counted (see Table 3). Each "VALID STOP" event that is accompanied by a coincidence with the solid state detector amplifier is a VALID EVENT, and is counted and analyzed by the system (see Table 3). Each valid event is formatted into a 32-bit long Pulse-Height-Analysis event whose contents are shown in Table 4. In addition to the TOF value and silicon detector Energy signal amplitude, each PHA event contains the two-bit TOF FLG from the DTOF analysis (see table notes). The gain of the amplifier used for the Energy signal is determined by the Logic Board, which examines the channel number for the high gain amplifier: if it is saturated (channel 2048, approximately $21 \mathrm{MeV}$ ) then the low gain channel number is used (maximum value approximately $174 \mathrm{MeV}$ ). The TOFERROR gives the status of one of the command bits (see below). The matrix box number is the result of the matrix rate calculation for this event, and is telemetered to allow verification of the on-board software calculation. There is also a 2-level priority that is stored along with the matrix rate number; this priority level is used to determine priority for telemetering this PHA event (see below).

SIT PHA events are formatted by the MISC; there are 11 PHA packets per minute allocated to SIT (APIDs 606-616), each with 64 PHA events for a total of 704 PHA events/minute. The first 704 events that occur during a minute are stored in a buffer in the MISC. If additional events occur and if their priority $=1$ (high), they will be written over existing events starting with the first event. This will continue until the number of events reaches a limit (LIMHI) that is set by ground command. After that no further events are written into the buffer. It should be noted that in the case of high event rates with many high priority events, this scheme will 
Table 3 SIT on-board rates: Matrix \& beacon rate assignments

\begin{tabular}{|c|c|}
\hline Rate & Description \\
\hline \multicolumn{2}{|c|}{ Discriminator rates } \\
\hline DR1 & START singles (prescaled by 8 ) \\
\hline DR2 & STOP singles \\
\hline DR3 & Valid Stop \\
\hline DR4 & SSD singles \\
\hline DR5 & Valid events (triple coincidence: START + STOP + SSD) \\
\hline DR6 & $\begin{array}{l}\text { Dead time counter: fraction of time when instrument was processing events } \\
(\times 32768)\end{array}$ \\
\hline DR7 & $\begin{array}{l}\text { Number of artificial STOP counts generated by DTOF to close windows } \\
\text { opened with a START pulse but followed by no STOP pulse within } 128 \mathrm{~ns} \\
\text { (prescaled by } 8 \text { ) }\end{array}$ \\
\hline DR8 & $\begin{array}{l}\text { TOF system error count (number of events with either TOF FLG } 1 \text { or } 2 \\
\text { non-zero) }\end{array}$ \\
\hline \multicolumn{2}{|l|}{ Matrix rates } \\
\hline MR1 & Priority 0 \\
\hline MR2 & Priority 1 \\
\hline MR3 & SSD high gain ramp \\
\hline MR4 & SSD low gain ramp \\
\hline MR5 & Discarded events (event FIFO not emptied) \\
\hline MR6 & Out of bounds (no matrix box found) \\
\hline MR7 & Junk (matrix box \#7) \\
\hline MR8-19 & H counts for different energy bins \\
\hline MR20-29 & ${ }^{3} \mathrm{He}$ counts $\left(+\right.$ some spillover from ${ }^{4} \mathrm{He}$ ) for different energy bins \\
\hline MR30-45 & ${ }^{4} \mathrm{He}$ counts for different energy bins \\
\hline MR46-62 & $\mathrm{C}$ counts for different energy bins \\
\hline MR63-78 & O counts for different energy bins \\
\hline MR79-94 & $\mathrm{Ne}+\mathrm{Mg}+\mathrm{Si}$ counts for different energy bins \\
\hline MR95-108 & Fe counts for different energy bins \\
\hline MR109-114 & $\mathrm{UH}(\mathrm{m}>80 \mathrm{amu})$ counts + background for different energy bins \\
\hline MR115-116 & Background \\
\hline \multicolumn{2}{|l|}{ Beacon rates } \\
\hline B1-4 & He counts \\
\hline B5-8 & $\mathrm{C}+\mathrm{N}+\mathrm{O}$ counts \\
\hline B9-12 & Fe counts \\
\hline
\end{tabular}

result in the first portion of the 704 PHA event buffer to be exclusively high priority events that had arrival times after the last event in the buffer.

\subsubsection{Rates}

In addition to the PHA events, SIT collects count-rate data described in Table 3. There are three general types of rates: discriminator rates which are outputs from the Energy and DTOF boards, and both matrix and beacon rates which are calculated in the MISC. The 8 discriminator rates have simple logic and are described in the table. Note that DR1 and DR7 are prescaled by a factor of 8 . Once per minute all the rates in the table are compressed from 
Table 4 SIT PHA event data

\begin{tabular}{lclll}
\hline Item & No. of bits & Bit location & Source $^{\mathrm{a}}$ & Comments \\
\hline TOF & 9 & $0(1 \mathrm{sb})-8$ & $\mathrm{~F}$ & Time of flight channel number \\
Energy & 11 & $9-19$ & $\mathrm{~F}$ & Solid state detector channel number \\
TOF FLG 1 & 1 & 20 & $\mathrm{~F}$ & TOF error flag 1 \\
TOF FLG $2^{\mathrm{b}}$ & 1 & 21 & $\mathrm{~F}$ & TOF error flag 2 \\
GAIN & 1 & 22 & $\mathrm{~F}$ & SSD energy gain bit $(0=$ high; $1=$ low $)$ \\
TOFERROR & 1 & 23 & $\mathrm{C}$ & Command bit state \\
Matrix box & 7 & $24-30$ & $\mathrm{~L}$ & Matrix box number \\
PRI & 1 & $31(\mathrm{msb})$ & $\mathrm{L}$ & Priority bit $(0=$ low; $1=$ high $)$ \\
\hline
\end{tabular}

a Data source: $\mathrm{F}=$ received from front-end logic/ACTEL; $\mathrm{C}=$ command state bit; $\mathrm{L}=\mathrm{Matrix}$ rate lookup table (low order 7 bits)

${ }^{\mathrm{b}}$ TOF flag encoding (bit 1, bit 2) shows discriminator firings during the TOF measurement period: $(0,0)=1$ start, 1 stop (nominal event); $(0,1)=n$ start $(n>1), 1$ stop; $(1,0)=1$ start, $n$ stop; $(1,1)$ $=n$ start, $n$ stop, or Stop occurred before start

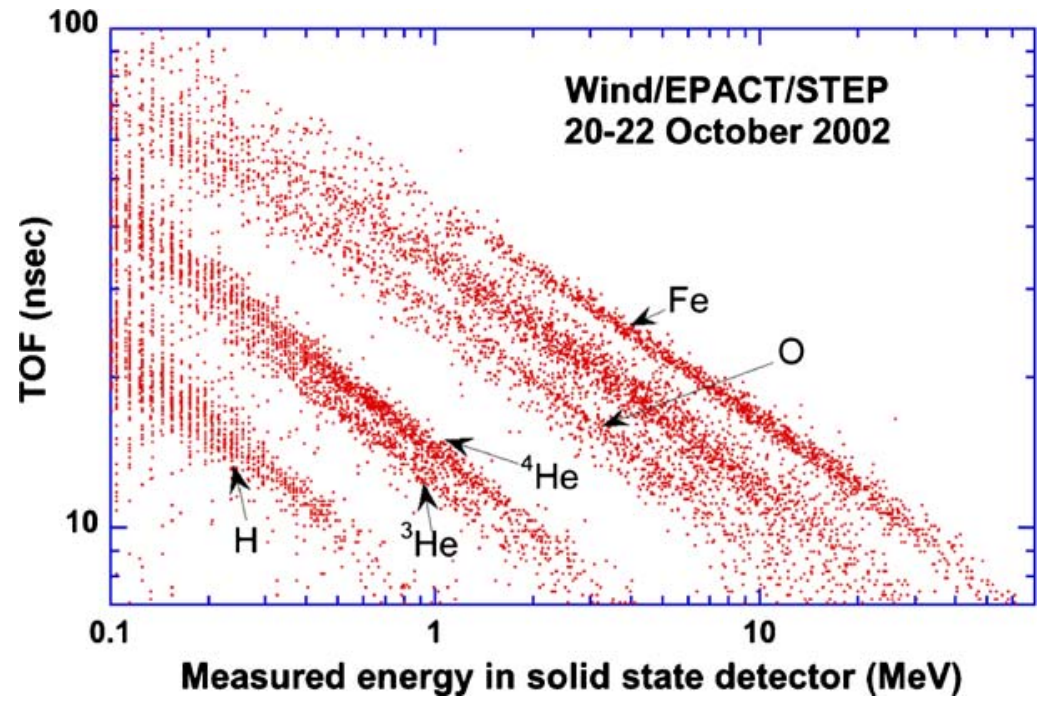

Fig. 16 Sample data from Wind/STEP 20-22 October $2002{ }^{3}$ He-rich period showing typical time vs. energy data from the telescope. Each dot represents a single ion event analyzed by the telescope

24 to 16 bits (for details, see Mewaldt et al., 2007), packetized in APID 605, and sent to SEP central.

The Matrix Rates and Beacon Rates are calculated in the MISC, and give particle intensities for several species allowing calculation of spectra with $60 \mathrm{~s}$ cadence when intensities are high. Figure 16 shows sample data from the Wind/STEP sensor that illustrates the appearance of data to be processed by SIT. The matrix rate calculation in essence picks box-like areas in the matrix, and sums together all the particles in one of these boxes. For example, the ${ }^{4} \mathrm{He}$ track would be split into a number of sections, and the number of events falling in each of these sections (boxes) would be counted separately. In defining the boxes, it is most convenient to transform the matrix shown in Figure 16 from time vs. energy to axes of mass vs. energy/nucleon, since the matrix boxes are defined in those terms. 
For the SIT telescope, the particle mass in AMU is reasonably approximated by:

$$
m=0.021^{*} E_{\mathrm{SSD}} \tau^{2}
$$

where $E_{\mathrm{SSD}}$ is the solid state detector signal in $\mathrm{MeV}$, and $\tau$ is the time of flight in nsec. The incident energy in $\mathrm{MeV} /$ nucleon $E_{\text {inc }}$ is approximately:

$$
E_{\mathrm{inc}}=\frac{1}{0.021 \tau^{2}}
$$

The mass-energy space covered by (2) and (3) is then scaled into a $128 \times 128$ matrix that is used for assigning the matrix boxes. The functions $f(m)$ and $f(E)$ that span this space are:

$$
f(m)=(\ln m+1)^{*}(128 / 7)
$$

and

$$
f\left(E_{\mathrm{inc}}\right)=\left(\ln \left(E_{\mathrm{inc}}\right)+5.5\right)^{*} 16 .
$$

If there were no defect or dead layers in the solid state detector, the particle distributions would follow constant $f(m)$ lines in the matrix. However, these effects are present and cause the tracks to sag below the constant $f(m)$ lines at low energies. The matrix box assignments for the different species take this into account, and so for each species the actual matrix boxes assigned deviate from the constant $f(m)$ lines at low energies. This can be seen in Figure 17, which shows a sample matrix of $f(m)$ vs. $f(E)$ with sample data from Wind/STEP (Figure 16) converted using Equations (2-5). Due to differences between the STEP and SIT solid-state detectors, the alignment of species areas and particle tracks is only approximate. The choice of 128 bins for the mass axis is based on the goal of separating $\mathrm{C}$ and $\mathrm{O}$ over most of the energy range, and for separating ${ }^{3} \mathrm{He}$ and ${ }^{4} \mathrm{He}$ over limited portions of the range. The choice of 128 bins for the energy axis is required to allow reasonably close matching between the nominal energy bins (whose start energies are spaced by a factor of $\sqrt{2}$ ) and the actual bins available on the $f(E)$ scale.

To carry out the calculations of Equations (2-5) in integer arithmetic, the MISC uses lookup tables that contain the logarithms of the solid state detector energy $(\mathrm{MeV})$ and timeof-flight (nsec) for each channel number. The $\left(f(m), f\left(E_{\text {inc }}\right)\right)$ cell address is used to look up a value in a $128 \times 128$ cell array whose contents are the matrix rate number assigned to that cell, the beacon rate number assigned to that cell, and the event priority level for that cell (high or low). Since the tables can be uploaded from the ground, the matrix rate, beacon rate, and priority determination can all be modified post launch if necessary. The colored bands in Figure 17 show sample assignments where matrix rate purple cells are $\mathrm{H}$, light blue are ${ }^{3} \mathrm{He}$, etc. Each colored band for a species is divided into $10-15$ different bands of $f\left(E_{\mathrm{inc}}\right)$ to give multiple energy points for particle spectra. The lookup tables require approximately 63 kbytes of memory, and the MISC processing algorithm can process events up to at least 4 $\mathrm{kHz}$, considerably higher than the maximum anticipated event rate of $\sim 1 \mathrm{kHz}$. The beacon rates are a subset of 12 rates for $\mathrm{He}, \mathrm{C}+\mathrm{N}+\mathrm{O}$, and $\mathrm{Fe}$ over wider energy bins than the matrix 


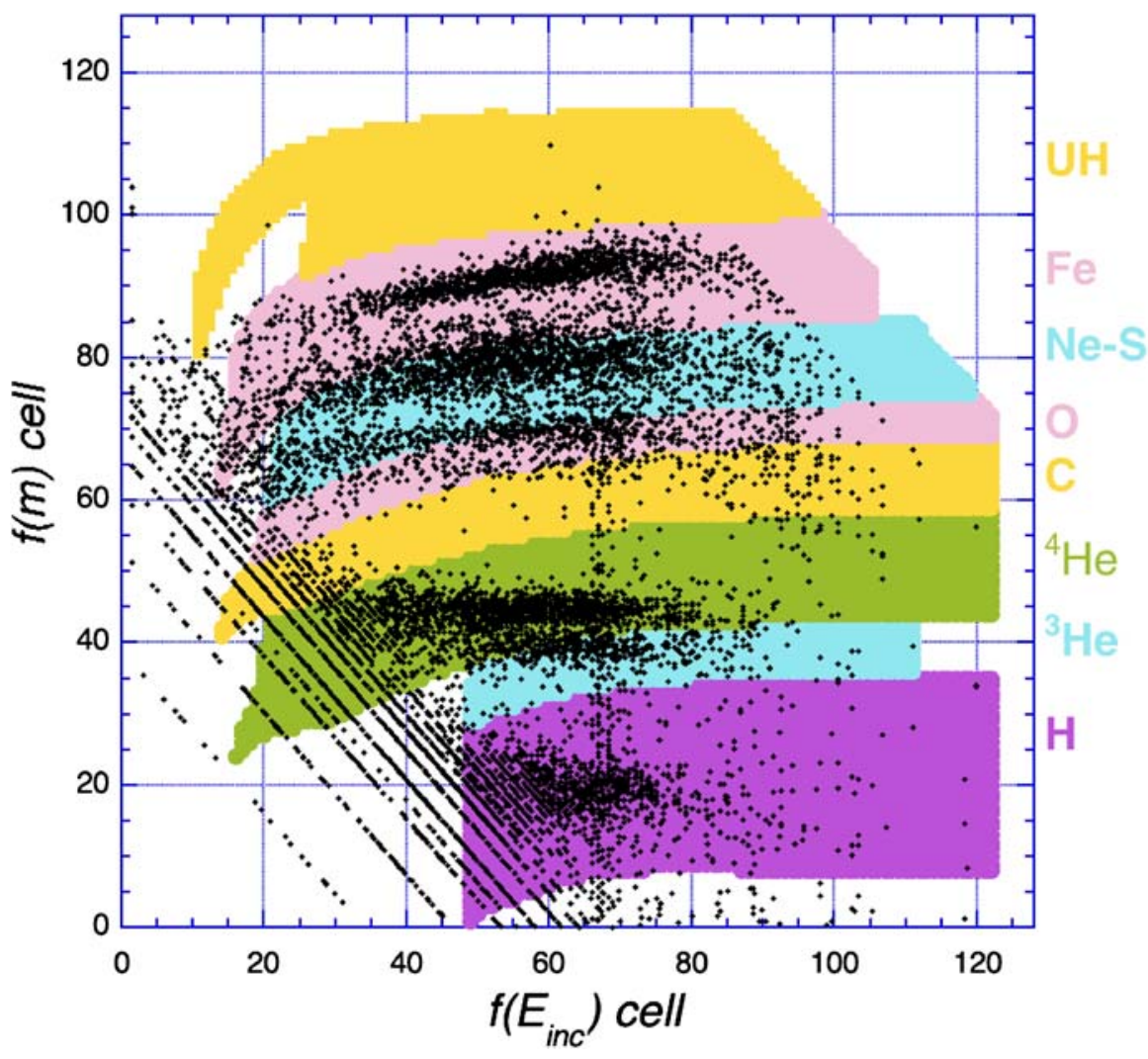

Fig. 17 Wind/STEP data from Figure 16 data converted to artificial mass and energy bins $\left(f(m)\right.$ and $\left.f\left(E_{\text {inc }}\right)\right)$ using Equations (2-5) and compared to nominal matrix box locations for SIT. Alignment between STEP and SIT data is only approximate. Species symbols on right correspond to the same color bands on the figure. Each species band is split into $10-15$ energy bins

rates. SIT beacon rates are combined with beacon rates from HET/LET in APID 624 and are part of the STEREO real-time telemetry stream.

Referring to Table 3, there are 116 Matrix Rates and 12 Beacon Rates. MR1-7 tally overall event rates, plus the lookup table rates. Note that MR6 is for events where the algorithm gave a value out of the range 1-128 for either $f\left(E_{\text {inc }}\right)$ or $f(m)$, and MR7 counts events that did not fall into an assigned species and energy bin.

\subsubsection{Calculation of Intensities}

The design of SIT is such that the detection efficiency for protons is $\sim$ few percent, and for helium $\sim 25 \%$ (these are the approximate expected initial values post-launch). For heavier ions, the detection efficiency is $\sim 1$. The omni-directional intensity $I_{j}$ for a single readout of Matrix Rate $j(=M R j)$ is

$$
I_{j}=\frac{1}{A \Omega \times \varepsilon_{j} \times \Delta E_{j} \times 60} M R_{j}
$$


where $I_{j}$ is in units of particles/( $\mathrm{s} \mathrm{cm}^{2} \mathrm{sr} \mathrm{MeV/nuc),A} \Omega$ is the geometry factor $\left(\mathrm{cm}^{2} \mathrm{sr}\right), \varepsilon_{j}$ is the efficiency for the species and energy box, $\Delta E_{j}$ is the energy interval covered by rate $M R_{j}$, and $M R_{j}$ is the number of counts in this rate bin for $60 \mathrm{~s}$. The calculation is analogous for the Beacon Rates. Due to the low H and He efficiency, the Wind/STEP data show that the triggering rate of the instrument will rarely exceed a few hundred counts/s, so the live time is $\sim 100 \%$.

Intensities may also be calculated for any arbitrary area of the $\tau$ vs. $E$ plane (Figure 16) using the PHA data. In this case, it is necessary to use the matrix rates to normalize the number of PHA counts because of the limited number of PHA events that are read out. For any arbitrary area in the $\tau$ vs. $E$ plane, let $N_{0 j}$ be the number of events with priority 0 , and $N_{1 j}$ be the number of events with priority 1 . Let $N_{0}$ and $N_{1}$ be the total number of events of priority 0 and 1 in the entire $\tau$ vs. $E$ plane. Then for a single $60 \mathrm{~s}$ period the intensity for the selected area is:

$$
I_{j}=\frac{1}{A \Omega \times \varepsilon_{j} \times \Delta E_{j} \times 60}\left(\frac{N_{0 j} \mathrm{MR} 1}{N_{0}}+\frac{N_{1 j} \mathrm{MR} 2}{N_{1}}\right)
$$

Note that MR1 and MR2 are the number of counts of priority 0 and 1 (Table 3). The values of $N_{0}$ and $N_{1}$ are obtained from the total group of PHA events transmitted for the same $60 \mathrm{~s}$ period as the matrix rate data. Longer time interval intensities are obtained by averaging the $60 \mathrm{~s}$ intensities.

\subsubsection{Particle Calibration Data \& Background Issues}

End-to-end testing of the SIT instruments was done by placing the units in vacuum and exposing them to alpha-sources. Figure 18 (left panel) shows a sample run from the AHEAD instrument just before it was mounted on the spacecraft. The mass resolution of $\sigma_{m} / m=0.1$ is sufficient to allow identification of impulsive SEP events with ${ }^{3} \mathrm{He} /{ }^{4} \mathrm{He}$ ratios above $\sim 10 \%$.
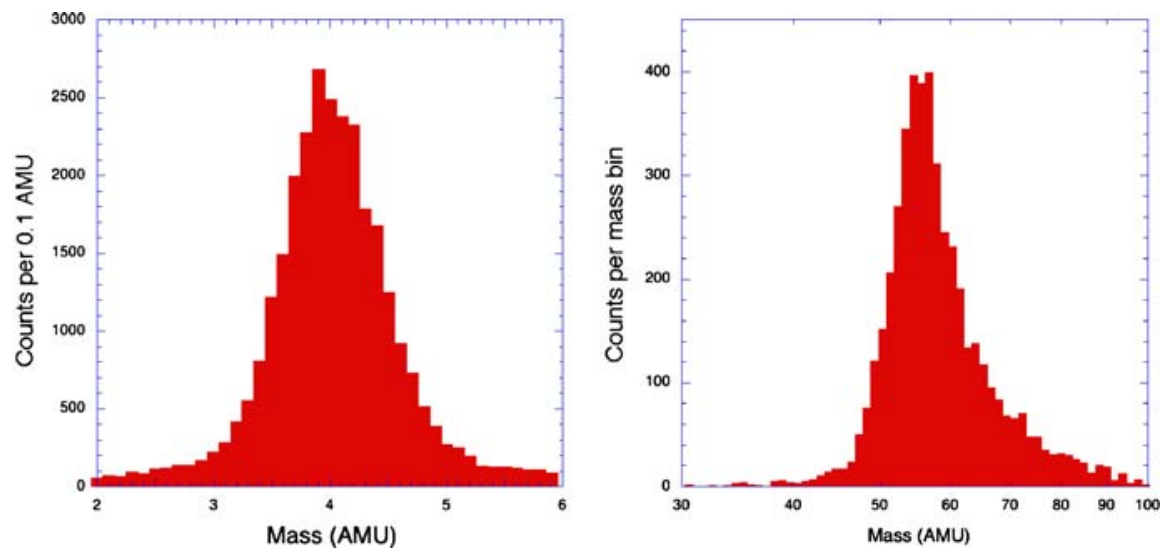

Fig. 18 STEREO/SIT calibration data samples. Left panel: alpha source resolution for the AHEAD flight instrument for particles with incident energy $0.7-0.9 \mathrm{MeV} / \mathrm{n}$, showing mass resolution $\sigma_{m} / m=0.1$. Right panel: Fe peak from engineering model telescope and AHEAD flight electronics for $\sim 0.25-0.5 \mathrm{MeV} /$ nucleon incident energy 
The instrument was also taken to an accelerator to test the electronics response for large pulse sizes and high event rates that could not be achieved with alpha sources. Figure 18 (right panel) shows a mass peak for Fe from this test, showing resolution that easily allows identification of e.g., Fe-rich solar particle events.

The sample Wind/STEP flight data shown in Figure 17 shows that background in the instrument is relatively low. The main limit on performance at low energies is caused by broadening of the mass peaks due to decreased accuracy in the solid state detector measurement. At high energies, dispersion of $\sim 1 \mathrm{~ns}$ in the TOF system leads to mass peak broadening; for example, at the $8 \mathrm{MeV} /$ nucleon, the time of flight is only about $2.5 \mathrm{~ns}$, and so the mass can be measured only approximately. There is no anti-coincidence detector in back of the SIT solid state detector, and so penetrating particles can trigger the system. However, for heavier ions, the penetration energy is so high (e.g. $15 \mathrm{MeV} / \mathrm{n}$ for $\mathrm{C}$ and higher energy/nucleon for heavier ions) that these events do not form an identifiable component of the background.

\section{Flight Operations}

SIT requires minimal commanding in flight. Early pointing maneuvers of the two STEREO spacecraft require that the telescope doors be kept closed early in the mission to avoid risk of the telescopes pointing toward the Sun. The door opening on STEREO Ahead is nominally scheduled for day 49 post-launch; for STEREO Behind it is scheduled for day 70 (the final door opening schedule depends on the exact launch date). After initial slow ramp-up of the high voltage SIT is fully operational. Due to the distinctive appearance of particle tracks in the $\tau$ vs. $E$ plane, the instrument calibration can be monitored easily, so there is no separate onboard calibration circuitry. It is anticipated that the matrix look-up tables will be adjusted after examining post-launch data; the provision for uploading new tables is included in the design. The only other anticipated commanding is adjustment of the MCP bias to compensate for loss of gain that can occur after extremely intense particle events such as those that occurred in 2000 July or 2003 October/November.

\subsection{Commands}

Table 5 lists commands used for routine operation of SIT. Additional commands such as turning on the instrument or loading new software or tables, are done through SEP Central and are not listed. Commands are normally executed by the MISC at the beginning of the next minute boundary so that command boundaries will coincide with the start of new rate and PHA accumulation intervals. However, if the command IMMED $=1$ has been sent, commands are executed without waiting for the minute boundary.

The command EONLY is a 1-bit command, with normal state 0. If EONLY $=1$, then events are analyzed with energy signal only (no TOF required). The command HVENABLE is a 1-bit command with turn on state $=0$, normal state $=1$ to allow operation of the $\mathrm{HV}$ supply. The command HVLEVEL is an 8-bit command that sets the voltage level of the HVPS. The command TOFERROR is a 1-bit command whose normal state is 0 , in which case PHA events that have either error flag set will be discarded. If TOFERROR $=1$, all PHA events are transmitted, regardless of the error flag values. JUNK is a 1-bit command whose normal state is 0 , and in this case PHA events with matrix box $=0$ (out of bounds) are not transmitted; if JUNK $=1$, all PHA events are transmitted regardless of their matrix box number. LIMHI is a 2-byte command that sets the limit of the number of high-priority PHA events that will be allowed to overwrite earlier events in the 704-event long PHA buffer. The 
Table 5 SIT command summary

\begin{tabular}{lll}
\hline Item & Description & Command name \\
\hline $\begin{array}{l}\text { Triggering logic } \\
\text { Coincidence requirement } \\
\text { Instrument operation }\end{array}$ & Enable/disable SSD coincidence & EONLY \\
High Voltage & Enable/disable HVPS & \\
High Voltage setting & Set High Voltage to level $x$ & HVENABLE \\
Software parameters & Enable/disable events with TOF error flags set & HLEVEL \\
PHA buffer contents & Enable/disable events with matrix box $=0$ & TOFERROR \\
Priority threshold & Set number of high priority PHA events that will & JUNK \\
& overwrite low priority events in PHA event buffer & \\
& (LIMHI) & \\
Lookup tables & Read in new lookup tables for calibration and matrix & (Multiple) \\
& boxes & \\
\hline
\end{tabular}

default value is LIMHI $=500$. The current setting of all these commands is transmitted once per minute in the Rate packet (APID 605).

Acknowledgements We are grateful to the many dedicated individuals whose efforts made possible the successful construction of the SIT instruments. At the University of Maryland Space Physics Group, we thank Tom James who carried out the original mechanical design of the telescope, and who inventoried the residual parts from Wind/STEP and organized the handoff of the mechanical design to GSFC. Scott Lasley ordered and tested the MCPs and provided support for the vacuum systems and testing/qualification for SIT. Joseph Dwyer participated in the early conceptual states of the on-board processing design, and suggested the pseudo-energy and mass scheme that was implemented. Jody Reed ably handled all aspects of the contracting and procurement initiation.

At the Max-Planck-Institut für Sonnensystemforschung (MPS), Lindau, Germany we thank the engineering staff for the construction of the ATOF, and particularly acknowledge the work of Klaus Heerlein on this project. The DTOF board was designed by Christian Dierker of the Institut für Datentechnik und Kommunikationsnetze at the Technische Universität Braunschweig. The flight ATOF and DTOF boards were constructed, and all flight qualifications were carried out by MPS.

Many individuals in the Goddard Space Flight Center Laboratory for High Energy Astrophysics supported the construction and qualification of SIT. The flight software was written by Kristin Wortman and Tom Nolan. George Winkert provided the Logic Board ACTEL FPGA which contained both the front-end logic and MISC. Kristin Wortman also provided critical support during many long hours of vacuum testing and spacecraft testing. Traci Pluchak-Rosnack assembled the flight electronics boards and telescope harness; Bert Nahory tested the solid state detectors; Haydar Teymourlouei supported the test data analysis. We thank John Hawk for the thermal design, and Mario Martins for untiring support of many cycles of thermal balance and thermal vacuum testing.

The Space Sciences Laboratory at the University of California Berkeley played a critical role in the development of SIT. Dave Curtis, IMPACT lead engineer, was actively involved throughout the project, supervising quality assurance and parts approval, and providing excellent tactical advice on innumerable issues. David Berg designed and built the SIT HVPS and housing, which was delivered fully qualified to the University of Maryland.

We thank Chuck Carlson and the operations crew of the Brookhaven Tandem Van de Graaff for their support during the August 2004 calibration of the prototype telescope and flight electronics.

The STEREO project office at Goddard Space Flight Center was unfailingly supportive throughout the project, and we especially thank Nick Chrissotimos for his leadership and Steve Wasserzug for energetic help with Goddard product assurance issues. We want to express our great appreciation to the IMPACT instrument manager, Lillian Reichenthal, whose hands-on involvement in support of SIT pre-delivery, and especially post-delivery was absolutely essential to the successful completion of the flight instruments. Finally we thank Richard Fisher, Eric Christian and Madhulika Guhathakurta, at NASA Headquarters for their enthusiastic support of the STEREO project and their dedication to maximizing the scientific returns of the mission.

This work was supported by the NASA STEREO project and in Germany by the Max-Planck-Gesellschaft zur Förderung der Wissenschaften and the Bundesministerium für Bildung und Forschung (BMBF) under grant 50 OC 0006. 


\section{References}

C.M.S. Cohen, E.C. Stone, R.A. Mewaldt, M.I. Desai, T.T. von Rosenvinge, M.E. Wiedenbeck, J. Geophys. Res. 110, A09S16 (2005), doi: 10.1029/2005JA011004

M.I. Desai, G.M. Mason, J.R. Dwyer, J.E. Mazur, R.E. Gold, S.M. Krimigis, et al., Astrophys. J. 588, 1149 (2003)

M.I. Desai, G.M. Mason, J.R. Dwyer, J.E. Mazur, C.W. Smith, R.M. Skoug, Astrophys. J. (Lett.) 553, L89 (2001)

M.I. Desai, G.M. Mason, R.E. Gold, S.M. Krimigis, C.M.S. Cohen, R.A. Mewaldt, et al., Astrophys. J. 649, 470 (2006a)

M.I. Desai, G.M. Mason, J.E. Mazur, J.R. Dwyer, Astrophys. J. (Lett.) 645, L81 (2006b)

M.I. Desai, G.M. Mason, M.E. Wiedenbeck, C.M.S. Cohen, J.E. Mazur, J.R. Dwyer, et al., Ap.J. 611, 1156 (2004)

D.C. Ellison, R. Ramaty, Astrophys. J. 298, 400 (1985)

A.B. Galvin, Ph.D. thesis, Dep't of Physics, University of Maryland (1982)

W.W. Harman, Fundamentals of Electronic Motion, (McGraw Hill, New York, 1953)

F.M. Ipavich, R.A. Lundgren, B.A. Lambird, G. Gloeckler, Nuc. Instr. Methods 154, 291 (1978)

S.W. Kahler, D.V. Reames, N.R. Sheeley Jr., Astrophys. J. 562, 558 (2001)

G. Li, G.P. Zank, W.K.M. Rice, J. Geophys. Res. 110, A06104 (2005), doi: 06110. 01029/02004JA010600

J. Luhmann et al., Space Sci. Rev. in press (2007), doi: 10.1007/s11214-007-9170-x

G.M. Mason, M.I. Desai, J.E. Mazur, J.R. Dwyer, in The Physics of Collisionless Shocks, Energetic Particles Accelerated in the Heliosphere: What is the Source Material? ed. by G. Li, G.P. Zank, C.T. Russell (AIP, New York, 2005), pp. 219-226

G.M. Mason, J.R. Dwyer, J.E. Mazur, Astrophys. J. (Lett.) 545, L157 (2000)

G.M. Mason, et al., Space Sci. Rev. 86, 409 (1998)

G.M. Mason, J.E. Mazur, J.R. Dwyer, Astrophys. J. (Lett.) 525, L133 (1999a)

G.M. Mason, J.E. Mazur, J.R. Dwyer, J.R. Jokipii, R.E. Gold, S.M. Krimigis, Astrophys. J. 606, 555 (2004)

G.M. Mason, J.E. Mazur, J.R. Dwyer, D.V. Reames, T.T. von Rosenvinge, Astrophys. J. (Lett.) 486, L149 (1997)

G.M. Mason, C.K. Ng, B. Klecker, G. Green, Astrophys. J. 339, 529 (1989)

G.M. Mason, T.R. Sanderson, Space Sci. Rev. 89, 77 (1999b)

G.M. Mason, et al., Astrophys. J. 574, 1039 (2002)

J.E. Mazur, G.M. Mason, J.R. Dwyer, J. Giacalone, J.R. Jokipii, E.C. Stone, Astrophys. J. (Lett.) 532, L79 (2000)

R.A. Mewaldt, C.M.S. Cohen, A.W. Labrador, R.A. Leske, G.M. Mason, M.I. Desai, et al., J. Geophys. Res. 110, A09S18 (2005), doi: 10.1029/2005JA011038

R.A. Mewaldt, C.M.S. Cohen, G.M. Mason, in Solar Eruptions and Energetic Particles, Geophysical Monograph\#165, ed. by N. Gopalswamy, R.A. Mewaldt, R.B. Torbert (AGU, Washington, 2006), pp. 115126

R.A. Mewaldt et al., in A.I.P. CP \#598, ed. by R.F. Wimmer-Schweingruber (A.I.P., New York, 2001), p. 165

R.A. Mewaldt, E.C. Stone, R.A. Leske, A.C. Cummings, M.E. Wiedenbeck, T.T. von Rosenvinge, Space Sci. Rev. in press (2007)

E. Möbius, M. Scholer, D. Hovestadt, B. Klecker, G. Gloeckler, Astrophys. J. 259, 397 (1982)

R. Müller-Mellin, S. Böttcher, J. Falenski, E. Rode, L. Duvet, T.R. Sanderson, et al., Space Sci. Rev. in press (2007)

M. Pick, G.M. Mason, Y.-M. Wang, C. Tan, and L. Wang, Astrophys. J. 648, 1247 (2006)

D.V. Reames, Space Sci. Rev. 90, 413 (1999)

D.V. Reames, Astrophys. J. (Lett.) 540, L111 (2000)

D.V. Reames, L.M. Barbier, T.T. von Rosenvinge, G.M. Mason, J.E. Mazur, J.R. Dwyer, Astrophys. J. 483, $515(1997 \mathrm{a})$

D.V. Reames, S. Kahler, C.K. Ng, Astrophys. J. 491, 414 (1997b)

I.G. Richardson, Planet. Space Sci. 33, 557 (1985)

I.G. Richardson, R.D. Zwickl, Planet. Space Sci. 32, 1179 (1984)

M. Shimojo, K. Shibata, Astrophys. J. 542, 1100 (2000)

L. Sollitt, Ph.D. thesis, Dep't. Physics, California Inst. of Tech. (2004)

L.S. Sollitt, E.C. Stone, R.A. Mewaldt, C.M.S. Cohen, R.A. Leske, M.E. Wiedenbeck, et al., ICRC 28, 3295 (2003)

A.J. Tylka, C.M.S. Cohen, W.F. Dietrich, M.A. Lee, C.G. Maclennan, R.A. Mewaldt, et al., Astrophys. J. 625, 474 (2005) 
A.J. Tylka, C.M.S. Cohen, W.F. Dietrich, C.G. Maclennan, R.E. McGuire, C.K. Ng, et al., Astrophys. J. (Lett.) 558, L59 (2001)

A.J. Tylka, M.A. Lee, Astrophys. J. 646, 1319 (2006)

P. Vandegriff, K. Wagstaff, G.C. Ho, J. Plauger, Adv. Space Res. 36, 2323 (2005)

T.T. von Rosenvinge et al., Space Sci. Rev. in press (2007)

T.T. von Rosenvinge et al., Space. Sci. Rev. 71, 155 (1995)

R. von Steiger, N.A. Schwadron, L.A. Fisk, J. Geiss, G. Gloeckler, S. Hefti, et al., J. Geophys. Res. 105, 27217 (2000)

L. Wang, R.P. Lin, S. Krucker, G.M. Mason, in Solar Wind 11/SOHO 16, Comparison between Impulsive ${ }^{3}$ He-rich Events and Energetic Electron Events (Whistler, Canada, 2005)

Y.-M. Wang, M. Pick, G.M. Mason, Astrophys. J. 639, 495 (2006)

M.E. Wiedenbeck, et al., in A.I.P. Conf. Proc. \#679, How Common are Flare Suprathermals in the Inner Heliosphere?, ed. by M. Velli, R. Bruno, F. Malara (A.I.P., New York, 2003), p. 652 